\title{
A TWO-SCALE FAILURE MODEL FOR HETEROGENEOUS MATERIALS: NUMERICAL IMPLEMENTATION BASED ON THE FINITE ELEMENT METHOD
}

\author{
S. Toro ${ }^{1,2}$, P.J. Sánchez ${ }^{1,2}$, A.E. Huespe ${ }^{1,3}$, S.M. Giusti ${ }^{4}$, P.J. Blanco ${ }^{5,6}$, R.A. Feijóo ${ }^{5,6}$ \\ ${ }^{1}$ CIMEC-UNL-CONICET, Güemes 3450, CP 3000, Santa Fe, SF, Argentina. \\ ${ }^{2}$ GIMNI-UTN, Lavaise 610, CP 3000, Santa Fe, SF, Argentina. \\ ${ }^{3}$ CIMNE, Technical Univ. of Catalonia, Campus Nord UPC, Edifici C-1, J.Girona 1-3, 08034 Barcelona, Spain. \\ ${ }^{4}$ GIDMA-UTN-FRC/CONICET, Maestro M. Lopez esq. Cruz Roja Argentina, CP X5016ZAA, Córdoba, Cba, \\ Argentina. \\ ${ }^{5}$ LNCC/MCTI Laboratório Nacional de Computação Científica, Getúlio Vargas 333, Petrópolis, Río de Janeiro, CEP: \\ 25651-075, Brasil. \\ ${ }^{6}$ INCT-MACC Instituto Nacional de Ciência e Tecnologia em Medicina Assistida por Computação Científica, Brasil.
}

Keywords: Multiscale formulations; Failure modeling of heterogeneous materials; Representative Volume Element $(R V E)$; Computational homogenization; Cohesive models.

\begin{abstract}
In the first part of this contribution, a brief theoretical revision of the mechanical and variational foundations of a Failure-Oriented Multiscale Formulation (FOMF) devised for modeling failure in heterogeneous materials is described.

The proposed model considers two well separated physical length scales, namely: (i) the "macro" scale where nucleation and evolution of a cohesive surface is considered as a medium to characterize the degradation phenomenon occurring at the lower length scale, and (ii) the "micro" scale where some mechanical processes that lead to the material failure are taking place, such as strain localization, damage, shear band formation, etc. These processes are modeled using the concept of Representative Volume Element $(R V E)$. On the macro scale, the traction separation response, characterizing the mechanical behavior of the cohesive interface, is a result of the failure processes simulated in the micro scale. The traction separation response is obtained by a particular homogenization technique applied on specific $R V E$ subdomains. Standard, as well as, Non-Standard boundary conditions are consistently derived in order to preserve "objectivity" of the homogenized response with respect to the micro-cell size.

In the second part of the paper, and as an original contribution, the detailed numerical implementation of the two-scale model based on the Finite Element Method is presented. Special attention is devoted to the topics which are distinctive of the FOMF, such as: (i) the finite element technologies adopted in each scale along with their corresponding algorithmic expressions, (ii) the generalized treatment given to the kinematical boundary conditions in the $R V E$ and (iii) how these kinematical restrictions affect the capturing of macroscopic material instability modes and the posterior evolution of failure at the $R V E$ level.

Finally, a set of numerical simulations is performed in order to show the potentialities of the proposed methodology, as well as, to compare and validate the numerical solution furnished by the two-scale model with respect to a mono-scale Direct Numerical Simulation (DNS) approach.
\end{abstract}




\section{Introduction}

Numerical simulation of heterogeneous materials using multiscale formulations is becoming a standard tool of analysis. In a multiscale context, the mechanical response of a macro-structural component can be retrieved through homogenization of complex micro-mechanical interactions taking place at the smaller length scales. This idea is particularly attractive to predict/comprehend the phenomenology involved in material failure scenarios, since the underlying heterogeneous micro-structural topology can play a decisive role in the activation and propagation of failure mechanisms at the macro scale level.

Although there are different starting points for developing multiscale approaches [3, 5, 9, 12, 15-18, 25, 39,45], Two-scale formulations ( "macro-micro") based on the concepts of Representative Volume Element $(R V E)$ and computational homogenization, sometimes referred to as $\mathrm{FE}^{2}$ models [10,11], have gained increasing popularity due to its intrinsic ability for modeling many classes of heterogeneous micro-structures: periodic, as well as, any statistical distribution of heterogeneities. Henceforth, we focus our developments on such "Two-scale RVE-based" theoretical framework. In this sense, the $R V E$ concept becomes a fundamental ingredient of the formulation.

Following a common trend in the literature, we introduce a rather standard definition of $R V E$ as the minimal micro-structural sample size from which the macroscopic homogenized response remains almost invariable $^{1}$, with respect to larger sample sizes. For micro-structures displaying a stable constitutive behavior, the existence of a physically admissible $R V E$-size is a well accepted notion. However, for heterogeneous micro-structures characterized by softening-based materials, recent contributions have proven that after crossing a critical point (i.e. during the macroscopic post-critical regime), an admissible $R V E$-size cannot be found in the context of conventional multiscale techniques [14, 28, 37,38]. New theories turn out to be necessary for modeling material failure phenomena within a multiscale paradigm [13]. Roughly speaking, there are two fundamental topics to be solved:

(I) how to introduce failure mechanisms in the macro scale model, such that they represent well the phenomenology captured by the microscale model;

(II) how to characterize this complex phenomenology via homogenization of micro-mechanical effects, keeping the theoretical foundations of well-posed multiscale formulations ( $R V E$ existence).

Point (I) could be tackled from different approaches. In the present contribution the cohesive interface method is adopted. The use of cohesive cracks for analyzing macroscopic material failure problems have been introduced in the pioneer works of Hillerborg [19], Barenblatt [1], Xu and Needleman [46]. Since then, numerous authors have followed this technique. Nowadays, cohesive models are widely used for representing quasi-brittle fracture, as well as ductile failure. An advantage of this methodology relies in the dramatic improvement of the kinematical description due to the introduction of displacement discontinuities, or strong discontinuities, according to the expected singular phenomenology provided by the physics of fracture. Cohesive interfaces have been utilized with a wide range of finite element techniques. Only to cite a few, we can mention the cohesive models defined between the finite element interfaces, such as advocated by Ortiz and coworkers [34], or cohesive interfaces which intersect arbitrarily the finite element mesh such as X-FEM or E-FEM techniques, see Oliver et al. [33] for a comparative analysis between the last two techniques.

On the other hand, a particularity of the cohesive interface method is related to the need of assuming a macroscopic traction-separation relation. This behavior can be difficult to characterize via phenomenological equations, specially for heterogeneous materials in general loading paths.

With reference to Point (II), and considering the cohesive models mentioned in the previous paragraphs, a natural option for characterizing the macro-cohesive response comes from a multiscale analysis jointly with computational homogenization. This methodological approach has been the subject of recent intensive research in the scientific community. A number of novel contributions have been made in this direction, see for example the approaches of Matous et al. [21], Verhoosel et al. [44], Nguyen et al. [27,29] Belytschko and coworkers [2,41], Geers and coworkers [6], Unger [43] and Souza et al. [42]. However, the development of a consistent method has remained full of major theoretical challenges.

Motivated by this open issue in material science, Sánchez and coworkers in $[37,38]$ have developed a multiscale model, called Failure-Oriented Multiscale Formulation (FOMF), based on axiomatic and rigorous variational foundations. The FOMF model provides two types of constitutive mechanical responses at the macroscale level in different situations: $(i)$ during the stable period, it determines a stress-strain relation derived from a classical homogenization procedure; and (ii) during the evolution of the cohesive

\footnotetext{
${ }^{1}$ Alternative definitions of the RVE concept can be found in the available literature, see [4] and references cited therein.
} 
macrocrack, it furnishes an homogenized mechanical response, i.e. a traction-separation relation, which is objective with respect to the size of the micro-cell adopted for the analysis.

In the present contribution, we advocate the use of the computational homogenization technique proposed by Sánchez et al. [37,38] which characterizes the mechanical response of the macro cohesive model, i.e. a traction-separation relation $(\boldsymbol{T} ; \boldsymbol{\beta})$, emerging from the microscale through a homogenization procedure. By adopting this approach, it is possible to tackle micro or mesoscopic failure mechanisms, developed at smaller length scale, as being the precursors of material failure at the macro scale. In particular, this work describes the most salient numerical and algorithmic issues that have been developed to implement the FOMF in the context of the finite element method. We address several issues of the micro-cell model implementation which are specific for multiscale analysis involving heterogeneous material failure problems. These specific issues are related to the use of a variational incremental framework including the possible change of the micro-cell boundary conditions during the mechanical evolution process. This key feature is exploited in strain localization problems.

Different boundary conditions on the RVE represent different multiscale sub-models. Then, with the generalized approach presented in this work, it is possible to simulate, in a flexible way, all these $R V E$ sub-models, as also a mixture of them or hybrid sub-models.

The paper is outlined as follows. Section 2 summarizes the FOMF approach by Sánchez et al. [37, 38]. Details about the numerical aspects and the proposed algorithms are shown in Sections 3, 4 and Appendix A, paying particular attention to the non-standard aspects that are out of the scope of the traditional multi scale methods. Section 3 describes the finite element models used for simulating the macro and micro scales. Section 4 presents the specific issues of the RVE model which have to be taken into account to get the complete degradation of the homogenized material. In Section 5 we present several numerical simulations. Finally, Section 6 is devoted to the conclusions.

\section{A two-scale material failure model}

The microscale analysis is performed by using a RVE to take into account the phenomenology induced by the degradation and strain localization phenomenon at the microscale. The crucial aspect is to get a $(\boldsymbol{T} ; \boldsymbol{\beta})$ relation at the coarse scale, that is independent of the cell size. An outline of this notion is sketched in Figure 1, where it is depicted that both domains, Cell 1 and $\mathrm{Cell}_{2}$, are valid cells to perform the stress homogenization analysis.

While the material response remains macroscopically stable, the existence of a RVE is a well established concept [26]. However, when the material becomes macroscopically unstable, its existence has recently been subjected to discussion. As proposed in the multiscale formulation of Sánchez et al. [37,38], the goal to find a RVE during the macroscopic unstable response can be achieved by modifying the information transfer procedure across both scales, as is summarized in the following subsections.

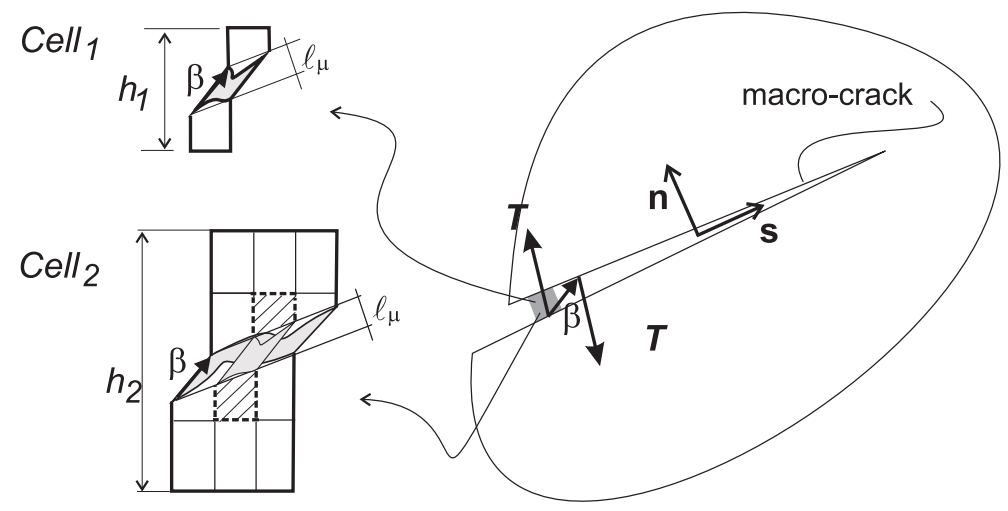

Figure 1: RVE's with different sizes that can be used to homogenize the cohesive traction vector defined on the crack surface.

\subsection{Model hypotheses.}

The basic assumptions of the model are described in the following items, and sketched in Figure 2:

i) Quasi-static problems are considered. The pseudo-time variable $t \in\left[0, t_{E}\right]$, where $\left[0, t_{E}\right]$ stands for the pseudo time interval of analysis, is used to account for the evolution of the non-linear material 
response. Increments of variables between the pseudo-time steps $(t-d t)$ and $(t)$ are denoted $(d(\cdot)=$ $\left.(\cdot)_{t}-(\cdot)_{t-d t}\right)$.

ii) The heterogeneous material response can be described by means of a model using two well-separated length scales. The structural scale, or macroscale, and the length scale in the order of the microconstituent sizes, or microscale. Infinitesimal strain theory is assumed for both scales.

iii) In the macroscale, the configuration of the body is denoted as $\Omega$ which is subjected to tractions $t^{*}$, in the boundary $\Gamma_{\sigma}$, and prescribed displacement $\boldsymbol{u}^{*}$, in $\Gamma_{u}$. Material points in $\Omega$ are denoted as " $\boldsymbol{x}$ ", and the prefix "macro-(•)" is used to indicate the fields defined at the macroscale, depending on $\boldsymbol{x}$. Every point $\boldsymbol{x} \in \Omega$ is linked to a microscale model through a heterogeneous RVE. The RVE domain is denoted $\Omega_{\mu}$ and their points are denoted $\boldsymbol{y}$. The prefix "micro-(•)" is alternatively used to denote the fields defined at the microscale, depending on $\boldsymbol{y}$.

iv) Initially, the macroscale is idealized as a statistically homogeneous continuum, with a heterogeneous microscale. At an arbitrary point $\boldsymbol{x}_{R}$ of this configuration, see Figure 2, the macro-displacement $\boldsymbol{u}$, macro-strain $\boldsymbol{\varepsilon}_{R}$, and the macro-stress $\boldsymbol{\sigma}$, characterize the mechanical state of the continuum medium. An alternative macro-mechanical scenario arises when a given critical condition, determined by micro-failure mechanisms, is reached. The new scenario is characterized in the model by introducing a macro-cohesive crack, as shown in the point $\boldsymbol{x}_{S}$ of Figure 2, where the existence of a cohesive force $\boldsymbol{T}$ being a function of the displacement jump $\boldsymbol{\beta}$ across the discontinuity surfaces is assumed. This cohesive force is determined through a specific homogenization technique.

v) In the microscale, we use a standard stress-strain approach for modeling the material failure. Then , the softening response is regularized by means of a smeared crack approach.

vi) In the variational formulation used in this work, it is defined an admissible kinematics which, after introducing the Hill-Mandel hypothesis, determines completely the homogenization rule that has to be used to compute the generalized stresses: $\boldsymbol{\sigma}$ in the points $\boldsymbol{x}_{R}$ and $\boldsymbol{T}$ in $\boldsymbol{x}_{S}$.

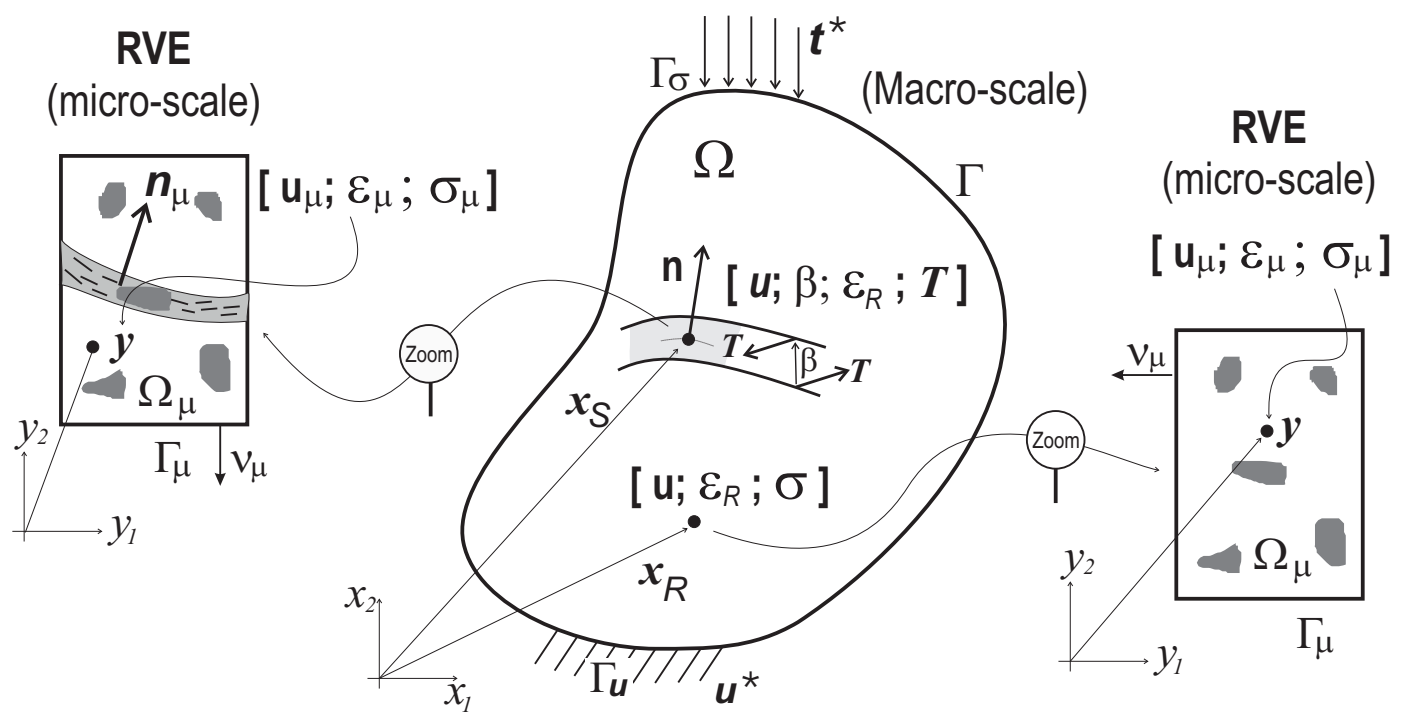

Figure 2: Basic ingredients of a two-scale model based on the RVE concept (macro-micro scale).

\subsection{Macroscopic model with strong discontinuity kinematics.}

According to the assumption (iii) in the previous Sub-Section, let us consider a body $\Omega$ in $\mathbb{R}^{\text {ndim }}$, with ndim being the dimension of the Euclidean space (in this work $n d i m=2$ ), undergoing a loading process, as shown in Figure 2.

During the loading process, we consider the situation when a macro crack $\mathcal{S}$, with a normal vector $\boldsymbol{n}$, arises in $\Omega$, as shown in Figure 3-a. The cohesive crack is mechanically characterized by the relative displacement jump $\boldsymbol{\beta}$ experienced by the surfaces of the crack, i.e. the crack opening, and the cohesive tractions $\boldsymbol{T}$ acting in those points. 
We follow the work of Simo et al. [40] to represent strong discontinuity kinematics for modeling the jump $\beta$ in the macroscale. Thus, we define an arbitrary sub-domain $\Omega^{\varphi}\left(\Omega^{\varphi} \subset \Omega\right)$ including the interface discontinuity $\mathcal{S}\left(\mathcal{S} \subset \Omega^{\varphi}\right)$ with smooth boundary: $\Gamma^{\varphi}\left(\Gamma^{\varphi}=\Gamma_{I}^{\varphi} \cup \Gamma_{I I}^{\varphi} \cup \mathcal{S}_{+} \cup \mathcal{S}_{-}\right)$as shown in Figure 3-b. Also, note that $\mathcal{S}$ divides $\Omega$ in two sub-domains: $\Omega_{+}$and $\Omega_{-}$with the normal vector $\boldsymbol{n}$ pointing toward $\Omega_{+}$.

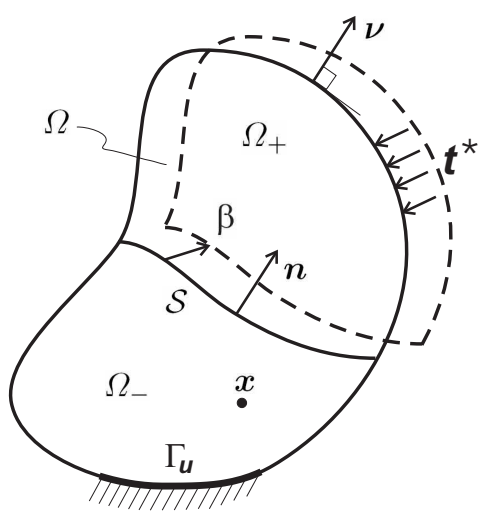

(a)

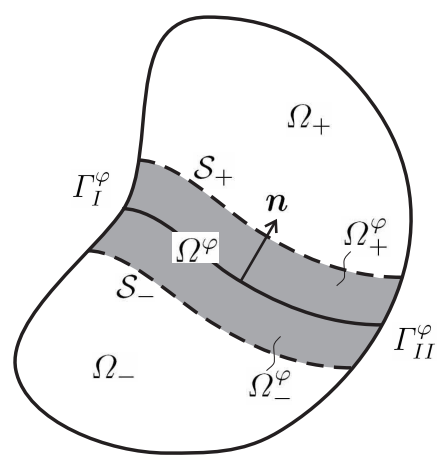

(b)

Figure 3: Mechanical problem exhibiting strong discontinuities across the material surface $\mathcal{S}$.

In this case, the mechanical response of $\Omega$ displaying a crack is described by the variational formulation of the problem summarized in Box 1, which has been taken from Sánchez et al. [37, 38]. Equation (1) describes the macro displacement field $\boldsymbol{u}$ exhibiting discontinuities, $\overline{\boldsymbol{u}}$ denotes a smooth field and $\mathscr{M}_{\mathcal{S}}(\boldsymbol{x})$ is the so-called Unit Jump function defined with the Heaviside step function, $\mathcal{H}_{\mathcal{S}}(\boldsymbol{x}): \Omega \rightarrow \mathbb{R}$, shifted to the discontinuity surface $\mathcal{S}: \mathcal{H}_{\mathcal{S}}(\boldsymbol{x})=0 \forall \boldsymbol{x} \in \Omega_{-}$and $\mathcal{H}_{\mathcal{S}}(\boldsymbol{x})=1 \forall \boldsymbol{x} \in \Omega_{+}$. The term $\varphi(x): \Omega \rightarrow \mathbb{R}$ is a sufficiently smooth, but otherwise arbitrary function, such that: $\varphi(\boldsymbol{x})=0 \forall \boldsymbol{x} \in \Omega_{-} \backslash \Omega_{-}^{\varphi}$ and $\varphi(\boldsymbol{x})=1$ $\forall \boldsymbol{x} \in \Omega_{+} \backslash \Omega_{+}^{\varphi}$. The displacement jump across $\mathcal{S}$ is denoted: $\boldsymbol{\beta}(\boldsymbol{x}, t)$.

The regular macro-strain $\boldsymbol{\varepsilon}_{R}$ is defined in points excluding $\mathcal{S}$, and is written in equation (2).

Hence, from (1) and (2), observe that the macro-displacement and macro-strain fields are completely described through the variables: $(\overline{\boldsymbol{u}}, \boldsymbol{\beta})$. The kinematically admissible macro-displacement set $\mathscr{U}$ is defined in (3). The associated virtual space of admissible variations $\mathscr{V}$ is given by equations (4) and (5). Fields in $\mathscr{V}$ are denoted with the symbol $(\hat{*})$.

Equation (6) describes, in a standard variational form, the macroscale equilibrium. Body forces $\boldsymbol{b}$ (per unit of volume) are considered to exist in $\Omega \backslash \mathcal{S}$, as well as cohesive tractions $\boldsymbol{T}$ acting on the macro-crack surfaces.

\subsection{Scale transition models.}

The item 4 in Box 1 summarizes the two-scale homogenization methodology defining the material response. This scheme distinguishes between regular points $\boldsymbol{x}_{R}$ in $\Omega / \mathcal{S}$ and singular points $\boldsymbol{x}_{S} \in \mathcal{S}$. In the first case, the homogenization technique determines $\sigma$ by means of the procedure described in Box 2 . We call this scheme, the Classical Multiscale Model (ClaMM). In fact, this procedure follows a standard model widely known in the literature (see Miehe et al. [24], Michel et al. [22], de Souza et al. [7,8], Peric et al. [35], and references cited therein). In the second case, item 4.2, the homogenization technique determines the tractions $\boldsymbol{T}$ as a function of the displacement jump $\boldsymbol{\beta}$ by means of the procedure described in Box 3 . This is called the Cohesive Multiscale Model (CohMM) and was recently proposed by Sánchez et al. [37,38]. The two models, ClaMM and CohMM, are shown in terms of generalized strains, stress and traction increments.

The criterion that defines when the macroscopic material response becomes unstable, and hence, when a macro-crack has to be introduced at a certain point $\boldsymbol{x}$, is defined in the Item 5 of Box 1 . This criterion is based on the singularity of the acoustic tensor $\boldsymbol{Q}$ derived from the homogenized tangent constitutive tensor $\boldsymbol{C}$, where: $d \boldsymbol{\sigma}=\boldsymbol{C}: d \boldsymbol{\varepsilon}_{R}$. The vectors $\boldsymbol{n}$ and $\boldsymbol{\gamma}$ are the eigenvectors of $\boldsymbol{Q}$ when it is singular. The vector $\boldsymbol{n}$ represents the normal vector to the macro crack $\mathcal{S}$ and $\gamma$ represents the instantaneous opening direction of the crack at $t_{N}\left(\left.\dot{\boldsymbol{\beta}}\right|_{t=t_{N}}\right)$. 


\section{1-Kinematics:}

$\boldsymbol{u}=\overline{\boldsymbol{u}}+\mathscr{M}_{\mathcal{S}} \boldsymbol{\beta} \quad ; \quad \mathscr{M}_{\mathcal{S}}(\boldsymbol{x}):=\mathcal{H}_{\mathcal{S}}(\boldsymbol{x})-\varphi(\boldsymbol{x}) \quad ; \quad \forall \boldsymbol{x} \in \Omega$

$\varepsilon=\varepsilon_{R}=\nabla_{x}^{s} \bar{u}+\mathscr{M}_{\mathcal{S}} \nabla_{x}^{s} \beta-\nabla_{x} \varphi \otimes^{s} \beta \quad ; \quad \forall x \in \Omega / \mathcal{S}$

$(\overline{\boldsymbol{u}}, \boldsymbol{\beta}) \in \mathscr{U} ; \mathscr{U} \equiv\left\{(\overline{\boldsymbol{u}}, \boldsymbol{\beta}) \mid \overline{\boldsymbol{u}} \in \mathbf{H}^{1}(\Omega), \boldsymbol{\beta} \in \mathbf{H}^{1}(\Omega)\right.$ and $\left.\left.\overline{\boldsymbol{u}}\right|_{\Gamma_{u}}=\boldsymbol{u}^{*}\right\}$

2-Virtual kinematically admissible actions (velocities):

$\hat{\boldsymbol{u}}=\hat{\overline{\boldsymbol{u}}}+\mathscr{M}_{S} \hat{\boldsymbol{\beta}}$

$(\hat{\overline{\boldsymbol{u}}}, \hat{\boldsymbol{\beta}}) \in \mathscr{V} ; \mathscr{V} \equiv\left\{(\hat{\overline{\boldsymbol{u}}}, \hat{\boldsymbol{\beta}}) \mid \hat{\overline{\boldsymbol{u}}} \in \mathbf{H}^{1}(\Omega), \hat{\boldsymbol{\beta}} \in \mathbf{H}^{1}(\Omega)\right.$ and $\left.\left.\hat{\overline{\boldsymbol{u}}}\right|_{\Gamma_{u}}=\boldsymbol{0}\right\}$

3-Variational equilibrium problem:

Given $\boldsymbol{b}$ and $\boldsymbol{t}^{*}$; find $(\overline{\boldsymbol{u}}, \boldsymbol{\beta}) \in \mathscr{U}$ such that:

$$
\begin{aligned}
\int_{\Omega \backslash S} \sigma \cdot \nabla_{\boldsymbol{x}}^{s}\left(\hat{\overline{\boldsymbol{u}}}+\mathscr{M}_{\mathcal{S}} \hat{\boldsymbol{\beta}}\right) d \Omega+\int_{\mathcal{S}} \boldsymbol{T} \cdot \hat{\boldsymbol{\beta}} d S- \\
\quad-\int_{\Omega \backslash \mathcal{S}} \boldsymbol{b} \cdot\left(\hat{\overline{\boldsymbol{u}}}+\mathscr{M}_{\mathcal{S}} \hat{\boldsymbol{\beta}}\right) d \Omega-\int_{\Gamma_{\sigma}} \boldsymbol{t}^{*} \cdot \hat{\overline{\boldsymbol{u}}} d \Gamma=0 \quad ; \quad \forall(\hat{\overline{\boldsymbol{u}}}, \hat{\boldsymbol{\beta}}) \in \mathscr{V}
\end{aligned}
$$

4-Required Constitutive responses via corresponding Multi-Scale Formulations:

$4.1 \forall x_{R} \in \Omega / \mathcal{S}$ and $\forall t \in\left[0, t_{E}\right]$ : Given $\varepsilon_{R t-d t}$ and $d \varepsilon_{R}$, find $d \sigma$,

via the ClaMM formulation (refer to Box 2)

Update: $\sigma=\sigma_{t-d t}+d \sigma$

4.2 $\forall \boldsymbol{x}_{S} \in \mathcal{S}$ and $\forall t \in\left[t_{N}, t_{E}\right]:$ Given $\left\{\boldsymbol{\varepsilon}_{R}, \boldsymbol{\beta}\right\}_{t-d t}$ and $\left\{d \boldsymbol{\varepsilon}_{R}, d \boldsymbol{\beta}\right\}$, find $d \boldsymbol{T}$,

via the CohMM formulation (refer to Box 3).

Update: $\boldsymbol{T}=\boldsymbol{T}_{t-d t}+d \boldsymbol{T}$

5-Cohesive crack nucleation criterion: (loss of material stability)

Find $\mathbb{S}_{N}=\left\{t_{N}, \boldsymbol{n}, \boldsymbol{\gamma}\right\}$, verifying the singularity of the Acoustic Tensor $\boldsymbol{Q}\left(\boldsymbol{\varepsilon}_{t}, \boldsymbol{n}\right)$ :

$\operatorname{det}\left(\boldsymbol{Q}\left(\boldsymbol{\varepsilon}_{t}, \boldsymbol{n}\right)\right)=0 \quad ; \quad \forall \boldsymbol{x} \in \Omega / \mathcal{S}$ and $\forall \boldsymbol{n} \in \mathbb{R}^{\text {ndim }}$

where $\boldsymbol{Q}\left(\boldsymbol{\varepsilon}_{t}, \boldsymbol{n}\right) \boldsymbol{\gamma}=\left[\left(\boldsymbol{C}\left(\boldsymbol{\varepsilon}_{t}\right) \boldsymbol{n}\right) \boldsymbol{\gamma}\right] \boldsymbol{n}, \forall \boldsymbol{\gamma} \in \mathbb{R}^{\mathrm{ndim}}$

and $\boldsymbol{C}\left(\boldsymbol{\varepsilon}_{t}\right)$ is the Homogenized Tangent Constitutive Tensor, obtained via the ClaMM formulation (refer to Box 2)

Box 1: Macro-mechanical problem with strong discontinuities. Basic concepts and ingredients. 


\subsubsection{Classical multiscale model (ClaMM).}

The stress determination in the points: $\boldsymbol{x} \in \Omega / \mathcal{S}$ is performed through a homogenization procedure using a RVE and adopting the framework proposed in de Souza et al. [7,8]. Box 2 summarizes this model.

Let $\Omega_{\mu}$ denote the RVE domain and $\Gamma_{\mu}$ its boundary with normal $v_{\mu}$, as shown in Figure 2. The terms: $\boldsymbol{u}_{\mu}, \boldsymbol{\varepsilon}_{\mu}$ and $\boldsymbol{\sigma}_{\mu}$ denote the micro-displacement, micro-strain and micro-stress fields in $\Omega_{\mu}$, respectively. Their increments, at the pseudo-time $t$, are denoted with $d(\bullet)$.

As usual in this kind of formulation, the incremental micro-strain field $d \boldsymbol{\varepsilon}_{\mu}$ is given by (see equation (13) in Box 2):

$$
d \boldsymbol{\varepsilon}_{\mu}=d \boldsymbol{\varepsilon}_{R}+\nabla_{y}^{s} d \tilde{\boldsymbol{u}}_{\mu}
$$

where the micro strain fluctuation increments is: $d \tilde{\boldsymbol{\varepsilon}}_{\mu}=\nabla_{y}^{s} d \tilde{\boldsymbol{u}}_{\mu}$ and $d \tilde{\boldsymbol{u}}_{\mu}$ is the micro displacement fluctuation increment field. It is interesting to mention here that in $[37,38]$ the authors present the Classical Multiscale Model introducing the concept of the "Insertion Operator". The generalization of this operator plays a fundamental role in the extension of this formulation for Failure-Oriented Multiscale Modeling. See [37,38] for more details about this very important theoretical aspect.

Equation (14) expresses that the volumetric average (in $\Omega_{\mu}$ ) of the micro-strain increment is equal to the macro-strain increment, or similarly, that the volumetric average of $d \tilde{\varepsilon}_{\mu}$ is zero, which is equivalent to constrain $d \tilde{\boldsymbol{u}}_{\mu}$, on the boundary $\Gamma_{\mu}$ (with normal vector $\boldsymbol{v}_{\mu}$ ), as follows:

$$
\int_{\Gamma_{\mu}} d \tilde{\boldsymbol{u}}_{\mu} \otimes^{s} \boldsymbol{v}_{\mu} d \Gamma_{\mu}=\mathbf{0}
$$

Prescription (8) characterize what is known as the Minimally Constrained Vector Space of kinematically admissible incremental displacement fluctuations at the RVE-level, $\tilde{\mathscr{U}}_{\mu}$ (see equation (15) in Box 2). The corresponding admissible variations of micro-displacement fluctuations vector space is denoted $\mathscr{V}_{\mu}$ (see equation (16) in Box 2).

It is noted that very well known (and used) multiscale models can be viewed as sub-models of the Minimum Kinematical Constraint Multiscale Model. They are considered sub-models because the kinematically admissible displacement fluctuation space, in each case, results a subspace of the Minimally Constrained vector space $\tilde{\mathscr{U}}_{\mu}$, i.e. they are particular cases of this general multiscale model. The considered kinematically admissible spaces of each sub-model are (omitting regularity requirements):

a) Taylor sub-model:

$$
\tilde{\mathscr{U}}_{\mu}^{T a y}=\mathscr{V}_{\mu}^{T a y}=\left\{\boldsymbol{\eta}_{\mu} \mid \boldsymbol{\eta}_{\mu}(\boldsymbol{y})=\mathbf{0} \quad \forall \boldsymbol{y} \in \Omega_{\mu}\right\}
$$

b) Linear boundary displacement sub-model:

$$
\tilde{\mathscr{U}}_{\mu}^{\text {Lin }}=\mathscr{V}_{\mu}^{\text {Lin }}=\left\{\boldsymbol{\eta}_{\mu} \mid \boldsymbol{\eta}_{\mu}(\boldsymbol{y})=\boldsymbol{0} \quad \forall \boldsymbol{y} \in \Gamma_{\mu}\right\}
$$

c) Periodic boundary fluctuation sub-model (the notation follows that of Miehe et al., [23]). Let us consider the RVE boundary $\Gamma_{\mu}$ that is partitioned into $\Gamma_{\mu}^{+}$and $\Gamma_{\mu}^{-}\left(\Gamma_{\mu}=\Gamma_{\mu}^{+} \cup \Gamma_{\mu}^{-}\right)$with unit normals vectors: $v_{\mu}^{+}$and $v_{\mu}^{-}$, respectively. Then, for every point $y_{i}^{+} \in \Gamma_{\mu}^{+}$there is the corresponding pair $\boldsymbol{y}_{i}^{-} \in \Gamma_{\mu}^{-}$, with the normal vectors: $\boldsymbol{v}_{\mu}\left(\boldsymbol{y}_{i}^{+}\right)=-\boldsymbol{v}_{\mu}\left(\boldsymbol{y}_{i}^{-}\right)$, which is associated to a given periodicity direction. Therefore, the periodic boundary condition satisfies: $d \tilde{\boldsymbol{u}}_{\mu}\left(\boldsymbol{y}_{i}^{+}\right)=d \tilde{\boldsymbol{u}}_{\mu}\left(\boldsymbol{y}_{i}^{-}\right)$and the corresponding admissible displacement spaces are:

$$
\tilde{\mathscr{U}}_{\mu}^{\text {Per }}=\mathscr{V}_{\mu}^{\text {Per }}=\left\{\boldsymbol{\eta}_{\mu} \mid \boldsymbol{\eta}_{\mu}\left(\boldsymbol{y}^{+}\right)=\boldsymbol{\eta}_{\mu}\left(\boldsymbol{y}^{-}\right) \quad \forall \operatorname{pair}\left\{\boldsymbol{y}^{-}, \boldsymbol{y}^{+}\right\}\right\}
$$

d) Minimum kinematical constraint model, as defined above, results when the expression (8) defines the kinematically and variationally admissible micro-displacement fluctuation spaces, as follows:

$$
\tilde{\mathscr{U}}_{\mu}^{M i n}=\mathscr{V}_{\mu}^{M i n}=\left\{\boldsymbol{\eta}_{\mu} \mid \int_{\Gamma_{\mu}} \boldsymbol{\eta}_{\mu} \otimes^{s} \boldsymbol{v}_{\mu} d \Gamma_{\mu}=\boldsymbol{0}\right\}
$$

The ClaMM satisfies the Hill-Mandel Variational Principle as a fundamental postulate (or hypothesis) of the formulation. This principle expresses that the internal virtual power in the macroscale, is equal to the volumetric average of the internal virtual power in the fine scale, and it is expressed in equation (18). From it, and after selecting specific virtual strains, two variational equations can be derived. The first 
Given $\boldsymbol{\varepsilon}_{R t-d t}$ and $d \boldsymbol{\varepsilon}_{R}$, find $d \boldsymbol{\sigma}$ and $\boldsymbol{C}$ :

1-Kinematics:

$d \boldsymbol{\varepsilon}_{\mu}=d \boldsymbol{\varepsilon}_{R}+\nabla_{\boldsymbol{y}}^{s} d \tilde{\boldsymbol{u}}_{\mu} \quad ; \quad \forall \boldsymbol{y} \in \Omega_{\mu}$

1.a-Kinematical Admissibility and Strain Homogenization Procedure:

$$
\begin{aligned}
& d \boldsymbol{\varepsilon}_{R}=\frac{1}{\left|\Omega_{\mu}\right|} \int_{\Omega_{\mu}} d \boldsymbol{\varepsilon}_{\mu} d \Omega_{\mu} \\
& d \tilde{\boldsymbol{u}}_{\mu} \in \tilde{\mathscr{U}}_{\mu} ; \tilde{\mathscr{U}}_{\mu} \equiv\left\{d \tilde{\boldsymbol{u}}_{\mu} \mid d \tilde{\boldsymbol{u}}_{\mu} \in \mathbf{H}^{1}\left(\Omega_{\mu}\right) \text { and } \int_{\Gamma_{\mu}} d \tilde{\boldsymbol{u}}_{\mu} \otimes^{s} \boldsymbol{v}_{\mu} d \Gamma_{\mu}=\boldsymbol{0}\right\}
\end{aligned}
$$

1.b-Virtual kinematically admissible actions (velocities):

$$
\begin{aligned}
& \hat{\boldsymbol{u}}_{\mu} \in \mathscr{V}_{\mu} ; \mathscr{V}_{\mu} \equiv\left\{\hat{\boldsymbol{u}}_{\mu} \mid \hat{\boldsymbol{u}}_{\mu} \in \mathbf{H}^{1}\left(\Omega_{\mu}\right) \text { and } \int_{\Gamma_{\mu}} \hat{\boldsymbol{u}}_{\mu} \otimes^{s} \boldsymbol{v}_{\mu} d \Gamma_{\mu}=\boldsymbol{0}\right\} \\
& \hat{\boldsymbol{\varepsilon}}_{\mu}=\hat{\boldsymbol{\varepsilon}}_{R}+\nabla_{\boldsymbol{y}}^{s} \hat{\boldsymbol{u}}_{\mu} \quad ; \quad \forall \hat{\boldsymbol{\varepsilon}}_{R} \text { and } \forall \hat{\boldsymbol{u}}_{\mu} \in \mathscr{V}_{\mu}
\end{aligned}
$$

2-Hill-Mandel Variational Principle of Macro-Homogenity:

$$
d \sigma \cdot \hat{\varepsilon}_{R}=\frac{1}{\left|\Omega_{\mu}\right|} \int_{\Omega_{\mu}} d \sigma_{\mu} \cdot \hat{\varepsilon}_{\mu} d \Omega_{\mu} \quad ; \quad \forall \hat{\varepsilon}_{R} \text { and } \forall \hat{\varepsilon}_{\mu} \text { kinematically admissible }
$$

2.a First consequence of the Hill-Mandel Variational Principle: Stress Homogenization

$$
d \sigma=\frac{1}{\left|\Omega_{\mu}\right|} \int_{\Omega_{\mu}} d \sigma_{\mu} d \Omega_{\mu}
$$

2.b Second consequence of the Hill-Mandel Variational Principle: Equilibrium problem at the micro-scale Given $\boldsymbol{\varepsilon}_{R t-d t}$ and $d \boldsymbol{\varepsilon}_{R}$; find $d \sigma_{\mu}$, such that:

$$
\int_{\Omega_{\mu}} d \sigma_{\mu} \cdot \nabla_{y}^{s} \hat{\boldsymbol{u}}_{\mu} d \Omega_{\mu}=0 \quad ; \quad \forall \hat{\boldsymbol{u}}_{\mu} \in \mathscr{V}_{\mu}
$$

3-Homogenized Tangent Constitutive Tensor:

$$
\boldsymbol{C}=\boldsymbol{C}_{T}+\tilde{\boldsymbol{C}}
$$

Taylor contribution

$$
\boldsymbol{C}_{T}=\frac{1}{\left|\Omega_{\mu}\right|} \int_{\Omega_{\mu}} \boldsymbol{C}_{\mu} d \Omega_{\mu}
$$

Fluctuation contribution

For $k, l=1,2,3$; find $\Delta \tilde{\boldsymbol{u}}_{k l} \in \mathscr{V}_{\mu}$ such that:

$\int_{\Omega_{\mu}} \boldsymbol{C}_{\mu} \nabla_{\boldsymbol{y}}^{s} \Delta \tilde{\boldsymbol{u}}_{k l} \cdot \nabla_{\boldsymbol{y}}^{s} \hat{\boldsymbol{u}}_{\mu} d \Omega_{\mu}=-\left[\int_{\Omega_{\mu}} \boldsymbol{C}_{\mu}\left(\boldsymbol{e}_{k} \otimes^{s} \boldsymbol{e}_{l}\right) \cdot \nabla_{\boldsymbol{y}}^{s} \hat{\boldsymbol{u}}_{\mu} d \Omega_{\mu}\right] \quad ; \quad \forall \hat{\boldsymbol{u}}_{\mu} \in \mathscr{V}_{\mu}$

$\tilde{\boldsymbol{C}}=\left[\frac{1}{\left|\Omega_{\mu}\right|} \int_{\Omega_{\mu}}\left(\boldsymbol{C}_{\mu}\right)_{i j p q}\left(\nabla_{\boldsymbol{y}}^{s} \Delta \tilde{\boldsymbol{u}}_{k l}\right)_{p q} d \Omega_{\mu}\right] \boldsymbol{e}_{i} \otimes \boldsymbol{e}_{j} \otimes \boldsymbol{e}_{k} \otimes \boldsymbol{e}_{l}$

Box 2: Classical Multi-scale Model (ClaMM). 
one, equation (19), defines the stress homogenization or stress transfer operator from the microscale to the macroscale. The second equation, given by expression (20), establishes the self-equilibrium of the microstress field. The stress $\sigma_{\mu}$ is connected with $\varepsilon_{\mu}$ through the microscopic constitutive model $\sigma_{\mu}\left(\varepsilon_{\mu}\right)$ and the constitutive tangent tensor $\boldsymbol{C}_{\mu}$ is such that: $d \sigma_{\mu}=C_{\mu} d \boldsymbol{\varepsilon}_{\mu}$.

The item 3 in Box 2 defines the homogeneized tangent constitutive tensor $\boldsymbol{C}$ obtained by deriving the expression $d \sigma_{\mu}(\boldsymbol{\varepsilon})$ given in (19). This tensor results from the addition of two terms: $i$ ) $\boldsymbol{C}_{T}$ is the volumetric average of the constitutive tangent tensor at the microscale $\boldsymbol{C}_{\mu}$, and $i$ ) the tensor $\tilde{\boldsymbol{C}}$, given by expression (23), that comes from the derivation of the displacement fluctuations. In (23), the vectors $\boldsymbol{e}$ denote the canonical basis of the Euclidean space. $\boldsymbol{C}$ is used to obtain the acoustic tensor, written in the Item 5 of Box 1, which singularity defines the macro-failure criterion.

\subsubsection{Cohesive multiscale model (CohMM).}

The homogenization of the traction-separation relation in $\boldsymbol{x}_{S} \in \mathcal{S}$, is performed through the Cohesive Multiscale Model (CohMM) proposed in [37,38] and summarized in the Box 3.

As shown in Box 1, see also Figure 2, the kinematics of the points $\boldsymbol{x}_{S}$, in $\mathcal{S}$, is characterized by the terms $\boldsymbol{\varepsilon}_{R}, \boldsymbol{\beta}$ and the normal vector to the discontinuity surface: $\boldsymbol{n}$. These kinematical variables are injected into the RVE using a specific strategy, such that the micro-strain increments are expressed by means of the equation (27) in Box 3. The first two terms in the right part of (27) represent the macro strain that is injected into the RVE:

$$
d \boldsymbol{\varepsilon}=d \boldsymbol{\varepsilon}_{R}+\phi_{\mu}^{L}(\boldsymbol{y}) \frac{d \boldsymbol{\beta} \otimes^{s} \boldsymbol{n}_{\mu}}{\ell_{M}} ; \quad \phi_{\mu}^{L}(\boldsymbol{y})= \begin{cases}\frac{\left|\Omega_{\mu}\right|}{\left|\Omega_{\mu}^{L}\right|}=\frac{\ell_{M}}{\ell_{\mu}} & \forall \boldsymbol{y} \in \Omega_{\mu}^{L} \\ 0 & \text { otherwise }\end{cases}
$$

where $\varepsilon_{R}$ is given by equation (2). While $d \varepsilon_{R}$ is uniformly distributed in the RVE, the collocation function $\phi_{\mu}^{L}(\boldsymbol{y})$ distributes the term: $\left(d \boldsymbol{\beta} \otimes^{s} \boldsymbol{n}_{\mu}\right) / \ell_{M}$ uniformly into the so-called RVE strain localization subdomain, $\Omega_{\mu}^{L}$, of the RVE, with $\Omega_{\mu}^{L} \subset \Omega_{\mu}$. In [37,38], $\Omega_{\mu}^{L}$ is defined as the RVE region where the material remains in a loading condition when the corresponding macroscopic point $\boldsymbol{x}$ first satisfies the instability criterion defined in Box 1, item 5, and a procedure is furnished to determine $\Omega_{\mu}^{L}$. The vector $\boldsymbol{n}_{\mu}$ is orthogonal to $\Omega_{\mu}^{L}$, such as shown in Figure 2. The parameter $\ell_{M}$ plays the role of a fictitious localization band width at the macroscale. Note also that, after replacing the function $\phi_{\mu}^{L}(y)$, the parameter $\ell_{M}$ is removed from the model and only remains the parameter $\ell_{\mu}$, which represents the width of the strain localized zone, $\Omega_{\mu}^{L}$, in the RVE. The parameter $\ell_{\mu}$ works as a characteristic length in the microscale model regularizing the material response during the unstable regime. The parameter $\ell_{\mu}$ plays an important role in determining the macroscale fracture energy of the homogenized material model.

We remark that the term: $\left(d \boldsymbol{\beta} \otimes^{s} \boldsymbol{n}_{\mu}\right) / \ell_{M}$ in (24), which is distributed in $\Omega_{\mu}^{L}$, is different from that proposed in $[37,38]$. Here, we use $\boldsymbol{n}_{\mu}$ instead of the normal vector $\boldsymbol{n}$ to the macro-crack. This proposal for injecting $d \boldsymbol{\beta}$ into the RVE is a key issue during the transition of models, from the ClaMM to the CohMM, to keep the kinematical consistency when micro-cracks with arbitrary directions are modeled in the RVE.

Finally, the third term in the right part of (27) corresponds to the micro-strain fluctuation increments.

The model also assumes that the volumetric averages of the micro-strain fluctuation increment in $\Omega_{\mu}$ and $\Omega_{\mu}^{L}$ are zero. These conditions are implicitly expressed in equations (28) and (29) of Box 3, and they are equivalent to writing the following two constraints on the incremental displacement fluctuation field:

$$
\begin{array}{lll}
\int_{\Gamma_{\mu}} d \tilde{\boldsymbol{u}}_{\mu} \otimes^{s} \boldsymbol{v}_{\mu} d \Gamma_{\mu}=\mathbf{0} & ; & (\mathrm{SBC}) \\
\int_{\Gamma_{\mu}^{L}} d \tilde{\boldsymbol{u}}_{\mu} \otimes^{s} \boldsymbol{v}_{\mu}^{L} d \Gamma_{\mu}=\mathbf{0} & ; & (\mathrm{NSBC})
\end{array}
$$

which characterize the space of admissible displacement fluctuation increments $\tilde{\mathscr{U}}_{\mu}^{L}$ in $\Omega_{\mu}$, as shown in expression (30) of Box 3. They also define the space $\mathscr{V}_{\mu}^{L}$ of kinematically admissible variations of macrodisplacement fluctuations $\hat{\boldsymbol{u}}_{\mu}$ given in expression (31). Expression (32) defines the micro-strain variations $\hat{\boldsymbol{\varepsilon}}_{\mu}$ that are kinematically admissible. In this expression, $\hat{\boldsymbol{\beta}}$ and $\hat{\boldsymbol{u}}_{\mu}$ are the admissible variations of displacement jumps and fluctuations, respectively. Selection of different subspaces of $\tilde{\mathscr{U}}_{\mu}^{L}$ and $\mathscr{V}_{\mu}^{L}$ similar to those given by expressions (9)-(11), furnishes different CohMM models.

The boundary condition (25) is called Standard Boundary Condition (SBC) because they also arises in the classical multiscale model (ClaMM), while (26) is called Non-Standard Boundary Condition (NSBC) and constitutes a new ingredient introduced by the present FOMF approach. 
Here, the homogenization of the traction vector $\boldsymbol{T} \in \mathcal{S}$, is derived from the Hill-Mandel Variational Principle given in equation (33). This principle expresses the balance between the internal virtual power at the macroscale, given by the product of the traction times the virtual displacement jump and divided by $\ell_{M}$, and the internal virtual power averaged in the microscale. From this variational principle, two consequences are derived: the first one is the equation (34), which defines the homogenization rule for $\boldsymbol{T}$; the second one provides the equation (35) representing, in a variational sense, the self-equilibrium of the micro-stress increments: $d \sigma_{\mu}$.

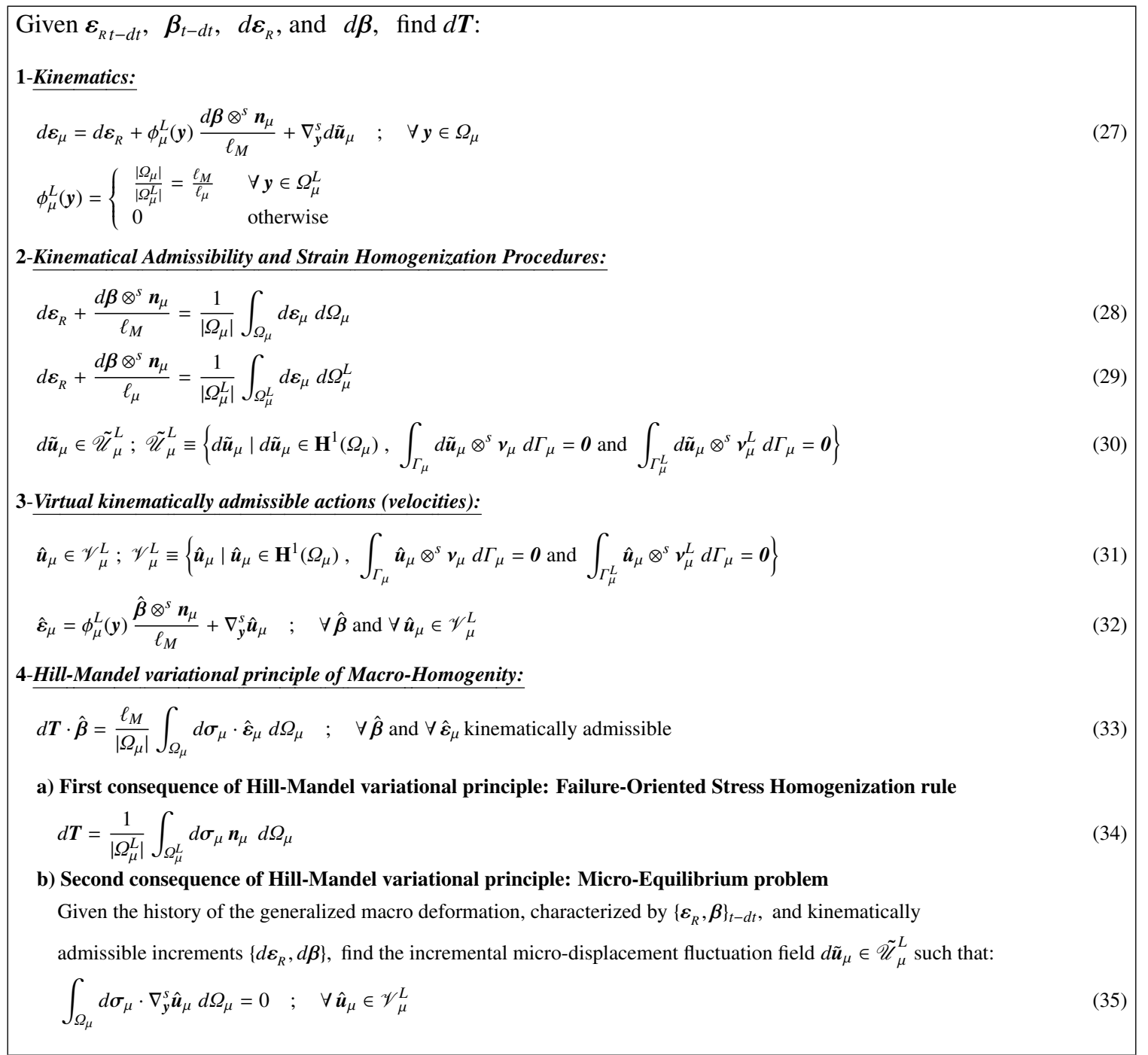

Box 3: Cohesive Multiscale Model (CohMM).

\section{Finite Element Models}

Given the particular characteristics of the FOMF approach, two different numerical techniques based on the Finite Element Method are adopted for simulating the macro and micro scales: $i$ ) an E-FEM technique for the macroscale, which utilizes strong discontinuity kinematics, and ii) a smeared crack approach with appropriate boundary conditions for the RVE simulation.

In the following, we describe some specific issues of both numerical models.

\subsection{Implementation of an E-FEM technique for the macroscale.}

A non-symmetric formulation with strong discontinuity kinematics, such as that proposed in [31] and denoted E-FEM technique, is here adopted. This formulation introduces additional displacement modes which specifically capture the discontinuities, or displacement jumps, arising in equation (1) of Box 1. 
Without loss of generality, we use the Constant Strain Triangle (CST) in plane states as the underlying finite element.

Let us consider a finite element mesh covering the body $\Omega$ having a displacement discontinuity surface $\mathcal{S}$. The surface divides the finite element mesh in two disjoint parts $\Omega_{+}$and $\Omega_{-}$, as shown in the Figure 4-b. The finite element interpolation of the displacement field, given in equation (1) of Box 1, can be written as:

$$
\boldsymbol{u}(\boldsymbol{x})=\sum_{i=1}^{n_{\text {node }}} N_{i}(\boldsymbol{x}) \boldsymbol{u}_{i}+\sum_{e=1}^{n_{\text {elem }}^{S}} \underbrace{\left(\mathcal{H}_{\mathcal{S}}^{e}(\boldsymbol{x})-N_{+}^{e}(\boldsymbol{x})\right)}_{\mathcal{M}_{\mathcal{S}}^{e}} \boldsymbol{\beta}^{e}
$$

where $n_{\text {node }}$ denotes the total number of nodes of the finite element mesh; $N_{i}$ and $\boldsymbol{u}_{i}$ are the standard shape function and the displacement vector (smooth part) of the $i-t$ node, respectively. $n_{\text {elem }}^{S}$ denotes the number of elements that are intersected by the discontinuity surface $\mathcal{S} ; \mathcal{H}_{\mathcal{S}}^{e}$ is the Heaviside step function of element $e$ shifted to $\mathcal{S}\left(\mathcal{H}_{\mathcal{S}}^{e}(\boldsymbol{x})=1\right.$ if $\boldsymbol{x} \in \Omega_{+}^{e}, \mathcal{H}_{\mathcal{S}}^{e}(\boldsymbol{x})=0$ otherwise $), N_{+}^{e}(\boldsymbol{x})$ denotes the shape function of the node located in the $\Omega_{+}$part of the element $e$, and $\beta^{e}$ is a constant displacement jump vector of the same element $e$. Being that the displacement jump is constant into the finite element, $\nabla \boldsymbol{\beta}^{e}$ is trivially zero.

Considering that the element $e$ is intersected by the discontinuity, the regular strain $\varepsilon_{R}^{e}$, given by equation (2) of Box 1, is interpolated as follows:

$$
\boldsymbol{\varepsilon}_{R}^{e}(\boldsymbol{x})=\boldsymbol{B}^{e}(\boldsymbol{x}) \boldsymbol{u}^{e}-\nabla_{\boldsymbol{x}}^{s} N_{+}^{e}(\boldsymbol{x}) \boldsymbol{\beta}^{e}
$$

where $\boldsymbol{B}^{e}(\boldsymbol{x})$ is the standard strain-displacement matrix. Using Voigt notation for tensors in $\mathbb{R}^{2}$, it can be written as:

$$
\boldsymbol{B}^{e}=\left[\begin{array}{cc|cc|cc}
\left(N_{1}^{e}\right)_{, x_{1}} & 0 & \left(N_{2}^{e}\right)_{, x_{1}} & 0 & \left(N_{3}^{e}\right)_{, x_{1}} & 0 \\
0 & \left(N_{1}^{e}\right)_{, x_{2}} & 0 & \left(N_{2}^{e}\right)_{, x_{2}} & 0 & \left(N_{3}^{e}\right)_{, x_{2}} \\
\left(N_{1}^{e}\right)_{, x_{2}} & \left(N_{1}^{e}\right)_{, x_{1}} & \left(N_{2}^{e}\right)_{, x_{2}} & \left(N_{2}^{e}\right)_{, x_{1}} & \left(N_{3}^{e}\right)_{, x_{2}} & \left(N_{3}^{e}\right)_{, x_{1}}
\end{array}\right]
$$

the first subindex of the shape functions $N^{e}$ expresses the element node number and the second subindex is the derivative respect to the corresponding coordinate. Adopting the same notation, the vector $\boldsymbol{u}^{e}$ is the element regular displacement vector:

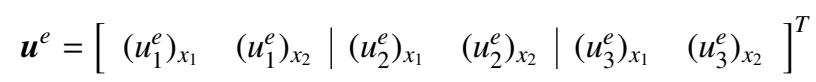

and $\nabla_{x}^{s} N_{+}^{e}$, as well as $\beta^{e}$ are:

$$
\nabla_{x}^{s} N_{+}^{e}=\left[\begin{array}{cc}
\left(N_{+}^{e}\right)_{, x_{1}} & 0 \\
0 & \left(N_{+}^{e}\right)_{x_{2}} \\
\left(N_{+}^{e}\right)_{, x_{2}} & \left(N_{+}^{e}\right)_{, x_{1}}
\end{array}\right] ; \quad \boldsymbol{\beta}^{e}=\left[\begin{array}{ll}
\beta_{x_{1}}^{e} & \beta_{x_{2}}^{e}
\end{array}\right]^{T}
$$

Introducing the interpolated displacements and strain fields, defined in (36) and (37), into the incremental equilibrium problem described in equation (6) of Box 1; and performing variations respect to $\boldsymbol{u}$ and $\boldsymbol{\beta}$ (both of these vectors collect all the element vectors $\boldsymbol{u}^{e}$ and $\boldsymbol{\beta}^{e}$ respectively), we can derive the following two incremental equilibrium equations:

$$
\begin{gathered}
\bigwedge_{e=1}^{n_{\text {elem }}} \int_{\Omega^{e} \backslash \mathcal{S}^{e}}\left(\boldsymbol{B}^{e}\right)^{T} d \boldsymbol{\sigma}^{e} d \Omega^{e}-\bigwedge_{e=1}^{n_{\text {elem }}}\left(d \boldsymbol{F}^{e}\right)^{e x t}=\mathbf{0} \\
-\int_{\Omega^{e} \backslash \mathcal{S}^{e}}\left[\nabla_{x}^{S}\left(N_{+}^{e}\right)\right]^{T} d \boldsymbol{\sigma}^{e} d \Omega^{e}+\int_{\mathcal{S}^{e}} d \boldsymbol{T}^{e} d \mathcal{S}^{e}=\mathbf{0}
\end{gathered}
$$

where $\bigwedge_{e=1}^{n_{\text {elem }}}$ denotes the assembling operator for the total number of finite elements: $n_{\text {elem }}$. The term $\left(d \boldsymbol{F}^{e}\right)^{\text {ext }}$ denotes the standard incremental external forces. The number of equations (40) is $n_{\text {elem }}^{S}$, one for every element intersected by $\mathcal{S}$.

An alternative non-symmetric formulation of this technique can be derived by replacing the incremental equilibrium equation (40) with a point wise collocation equation, per element, as follows (see [32]):

$$
-d \boldsymbol{\sigma}^{e} \boldsymbol{n}^{e}+d \boldsymbol{T}^{e}=\mathbf{0}
$$

where $d \boldsymbol{\sigma}^{e}$ is evaluated in a representative point of $\Omega^{e} \backslash \mathcal{S}^{e}$, and $\boldsymbol{n}^{e}$ is the unit vector normal to the cohesive interface $\mathcal{S}^{e}$ in the element, as shown in Figure 4-b. 
By using triangular elements as mentioned above, the enrichment shape function $\mathcal{M}_{\mathcal{S}}$, in (36), looks like that depicted in Figure 4-a. The numerical integration of expression (39) and (41) is performed by means of two quadrature points. Figure 4-b depicts the integration points named PGR and PGL.

The integration point PGR (square-symbol in Figure 4-b) is related to the domain $\Omega^{e} \backslash \mathcal{S}^{e}$, and thus, it is used to evaluate the regular component of the strain $\varepsilon_{R}^{e}$, equation (37), and the integrals in (39). Alternatively, the point PGL (x-symbol in Figure 4-(b)) is related to the domain $\mathcal{S}^{e}$. Both points, PGR and PGL, are used to evaluate the traction continuity condition (41) across the discontinuity surface $\mathcal{S}^{e}$, as follows:

$$
\underbrace{d \sigma^{e}}_{P G R} n^{e}=\underbrace{d \boldsymbol{T}^{e}}_{P G L}
$$

Each Gauss point is linked to its corresponding RVE. Prior to the macroscopic bifurcation, both RVE's evolve identically, since a constant regular-strain triangle is adopted. Thus equation (42) is trivially fulfilled. During the loading process and after crossing the bifurcation point, each RVE representing the PGR and PGL points evolves following different branches, as it is explained next.

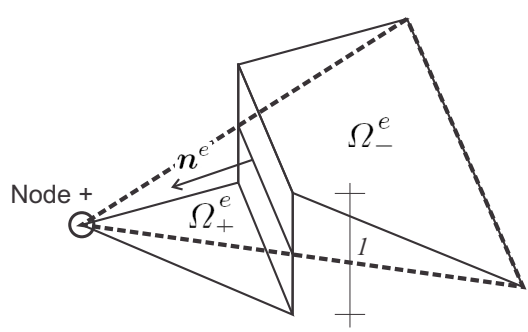

(a)

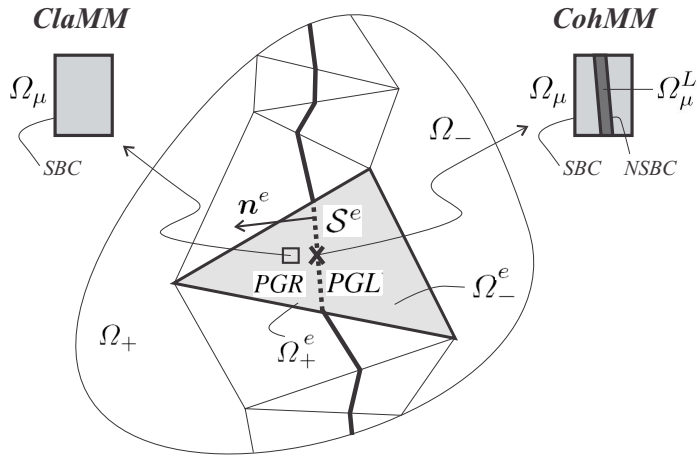

(b)

Figure 4: Finite element technique with embedded strong discontinuities in the macroscale: (a) shape function for the enhanced discontinuous mode; (b) a body discretized with a finite element mesh using the E-FEM methodology.

The Classical Multiscale Model (ClaMM), defined in Sub-Section 2.3.1 and Box 2, is used for the stress homogenization procedure in the point PGR, during the complete loading history. Thus, the corresponding incremental strain, $d \varepsilon_{R}$, is homogeneously inserted into the RVE and the increment of the homogenized stress, $d \sigma$, is obtained. After detecting the macro-bifurcation condition, an incremental elastic response is enforced in every point of this RVE, constraining the regular Gauss point to behave as a homogenized elastic, but possibly degraded, model. Also, when the bifurcation condition is fulfilled, the Cohesive Multiscale Model defined in Sub-Section 2.3.2 and Box 3 is used for homogenizing the traction increment in the point PGL. The incremental generalized kinematics, characterized by $d \boldsymbol{\varepsilon}_{R}, d \boldsymbol{\beta}$ and $\boldsymbol{n}^{\boldsymbol{e}}$, is inserted into the RVE using the equation (27) of Box 3. Note that both, the Standard as well as the Non-Standard Boundary Conditions (SBC and NSBC) are prescribed in this RVE. It is of crucial importance to prescribe them in incremental (rate) form. The increment of the homogenized traction vector, $d \boldsymbol{T}$, is then obtained by using the generalized homogenization formula given by expression (34).

After assembling the expressions in (39) and considering (41), $\boldsymbol{u}$ and $\boldsymbol{\beta}$ can be found by solving the non-linear system of equations:

$$
\mathcal{R}(\boldsymbol{u}, \boldsymbol{\beta})=\left[\begin{array}{l}
\mathcal{R}_{u} \\
\mathcal{R}_{\beta}
\end{array}\right]=\mathbf{0}
$$

where $\mathcal{R}_{\boldsymbol{u}}$ and $\mathcal{R}_{\boldsymbol{\beta}}$ are the residue (left term) of equations (39) and (41), respectively.

\subsubsection{Determination of the bifurcation condition at the macroscale.}

At the macroscale level and after convergence of the Newton-Raphson iterative procedure, the bifurcation condition expressed in the item 5 of Box 1 is evaluated as follows. The homogenized constitutive tangent tensor, $\boldsymbol{C}$, is determined in every quadrature point, and thus we can compute $\boldsymbol{Q}$. Then, using a swept algorithm, we find the minimum value:

$$
\omega=\min _{\theta=1: \Delta \theta: \pi} \operatorname{det}(\boldsymbol{Q}(\boldsymbol{n}(\theta)))
$$


by typically predefining an angle increment: $\Delta \theta$. When the condition $\omega \leq 0$ is found for the first time and for some $\theta$, the flag indicating the bifurcation state of that quadrature point is set to "TRUE" (the nucleation time $t_{N}$ is obtained). In general, problem (44) furnishes two solutions $\left(\theta_{\boldsymbol{n}}, \theta_{\gamma}\right)$ which define the normal vector to the crack $\boldsymbol{n}$, as well as $\boldsymbol{\gamma}$.

\subsection{Implementation of the FEM technique for the microscale.}

A standard finite element method is proposed for evaluating the numerical solution of the micro-cell problem evolution. Let us consider a micro-cell finite element mesh where the usual interpolation of the displacement fluctuation field $\tilde{\boldsymbol{u}}(\boldsymbol{y})$ is:

$$
\tilde{\boldsymbol{u}}_{\mu}(\boldsymbol{y})=\sum_{i=1}^{n_{\text {node }}^{\mu}} N_{i \mu}(\boldsymbol{y}) \boldsymbol{q}_{i}
$$

where $N_{i \mu}(\boldsymbol{y})$ is the standard shape function of the finite element basis corresponding to node $i$ and $\boldsymbol{q}_{i}$ is the nodal value of the displacement fluctuation for the same node; $n_{\text {node }}^{\mu}$ is the total number of nodes in the RVE finite element mesh. The vector collecting the displacement fluctuations of all nodes is denoted $\boldsymbol{q}$ and its dimension is $n=2 n_{\text {node }}^{\mu}$ (without loss of generality, we are considering problems in $\mathbb{R}^{2}$ with two d.o.f.'s per node). Then, $n$ is the total number of d.o.f.'s of the discretized problem.

As it is shown in Sub-Section 2.3.1, and afterward generalized to the CohMM approach, different RVE sub-models are defined depending on the functional spaces $\tilde{\mathscr{U}}_{\mu}$ and $\mathscr{V}_{\mu}$ from where the displacement solution is drawn. In a similar way, the increment of the discrete micro-cell nodal displacement fluctuation vector $\Delta \boldsymbol{q}$ can be searched in different vectorial spaces. Let us denote $\tilde{\mathscr{U}}_{q}$ the finite dimensional vectorial space of admissible nodal displacements, where $\left(\Delta \boldsymbol{q} \in \tilde{\mathscr{U}}_{q}\right)$, and $\mathscr{V}_{q}$ denotes the admissible variations of displacement fluctuation $\hat{\boldsymbol{q}}\left(\hat{\boldsymbol{q}}=\Delta \boldsymbol{q}_{2}-\Delta \boldsymbol{q}_{1}\right.$ with $\left.\Delta \boldsymbol{q}_{2}, \Delta \boldsymbol{q}_{1} \in \tilde{\mathscr{U}}_{q}\right)$. In correspondence with expressions (9)-(11) defining different RVE sub-models, we identify the discrete spaces:

a) Taylor sub-model:

$$
\tilde{\mathscr{U}}_{q}^{\text {Tay }}=\mathscr{V}_{q}^{\text {Tay }}=\{\Delta \boldsymbol{q} \mid \Delta \boldsymbol{q}=\mathbf{0}\}
$$

b) Linear boundary displacement sub-model;

$$
\tilde{\mathscr{U}}_{q}^{\text {Lin }}=\mathscr{V}_{q}^{\text {Lin }}=\left\{\Delta \boldsymbol{q} \mid \Delta \boldsymbol{q}_{i}=\boldsymbol{0} ; \quad \forall \text { node } i \in \Gamma_{\mu}\right\}
$$

c) Periodic sub-model: considering the RVE boundary $\Gamma_{\mu}$ partitioned in $\Gamma_{\mu}^{+}$and $\Gamma_{\mu}^{-}$, such that, for every nodal point $\boldsymbol{y}_{i}^{+} \in \Gamma_{\mu}^{+}$there is the corresponding point $\boldsymbol{y}_{i}^{-} \in \Gamma_{\mu}^{-}$, lying along the periodicity direction. With this partition of $\Gamma_{\mu}$ at hands, two normal vectors to the external boundary, in opposite sides, are given by: $\boldsymbol{v}_{\mu}\left(\boldsymbol{y}_{i}^{+}\right)=-\boldsymbol{v}_{\mu}\left(\boldsymbol{y}_{i}^{-}\right)$. This model satisfies the constraint: $\Delta \tilde{\boldsymbol{u}}_{\mu}\left(\boldsymbol{y}_{i}^{+}\right)=\Delta \tilde{\boldsymbol{u}}_{\mu}\left(\boldsymbol{y}_{i}^{-}\right)$. Then, the admissible displacement fluctuation spaces can be written as follows:

$$
\tilde{\mathscr{U}}_{q}^{\text {Per }}=\mathscr{V}_{q}^{\text {Per }}=\left\{\Delta \boldsymbol{q} \mid \Delta \boldsymbol{q}_{i}^{+}=\Delta \tilde{\boldsymbol{u}}_{\mu}\left(\boldsymbol{y}_{i}^{-}\right) ; \quad \forall \text { node } i+\in \Gamma_{\mu}^{+}\right\}
$$

where the constraint: $\Delta \boldsymbol{q}_{i}^{+}=\Delta \tilde{\boldsymbol{u}}_{\mu}\left(\boldsymbol{y}_{i}^{-}\right)$, is introduced for every node $i^{+}$in $\Gamma_{\mu}^{+}$. Note that, we do not require, a-priori, the presence of a nodal point in the position $y_{i}^{-}$, where the displacement fluctuation interpolation is constrained to have the same displacement as the node $i^{+}$.

Next, the periodic sub-model is generalized in the sense that the periodicity direction can be arbitrarily defined. In this case, we identify the associated pair of boundary points $\left(\boldsymbol{y}_{i}^{+}, \boldsymbol{y}_{i}^{-}\right)$, such that, they are the intersection points between the parallel line to the periodicity direction and the boundary surface $\Gamma_{\mu}$.

d) Minimum kinematical constraint model :

$$
\tilde{\mathscr{U}}_{q}^{\text {Min }}=\mathscr{V}_{q}^{\text {Min }}=\left\{\Delta \boldsymbol{q} \mid \int_{\breve{\Gamma}} \sum_{i}\left(N_{i \mu}(\boldsymbol{y}) \Delta \boldsymbol{q}_{i} \otimes^{s} \boldsymbol{v}_{i \mu}\right) d \breve{\Gamma}=\boldsymbol{0} ; \quad \forall \text { node } i \in \breve{\Gamma}\right\}
$$

the integration domain $\breve{\Gamma}$ depends of the model adopted during the analysis. Thus, in the ClaMM formulation, the constraint (8) (SBC), which prescribes the displacement fluctuation increments, involves an integration surface $\breve{\Gamma}$ coinciding with the RVE external boundary: $\breve{\Gamma} \equiv \Gamma_{\mu}$. In the CohMM formulation, the constraint (25) also involves an identical integration domain: $\breve{\Gamma} \equiv \Gamma_{\mu}$. However, an additional equation (49) (NSBC) shall be prescribed with the integration domain given by: $\breve{\Gamma} \equiv \Gamma_{\mu}^{L}$, as shown in equation (26). 
Hence, the change of model during the course of analysis, from the ClaMM to the CohMM, requires a change of the RVE boundary conditions.

Even when the ClaMM can be defined using one of the standard spaces described in the items $(a)-(d)$ above, the CohMM requires a hybrid combination of them.

In the following Sub-Sections, we present a systematic algorithm to manage, in an unified and flexible format, a wide range of constraints that can be imposed to the vector $\Delta \boldsymbol{q}$. The numerical procedure is based on an exact enforcement of these constraints, by performing a partition of the total d.o.f.'s of the vector $\Delta \boldsymbol{q}$, and a subsequent static condensation of one of these partitions.

\subsubsection{Numerical treatment given to the kinematical constraints.}

The different kinds of micro-cell sub-models, and the respective kinematical constraints prescribing the increments of the nodal displacement fluctuations, are characterized by four generic situations. They are described in the following items.

Case i) Nodes with the increments of displacement fluctuations prescribed to zero.

Assuming that there are " $n_{c}$ " d.o.f.'s with a kinematical constraint of the type: $\Delta q_{j}=0$, where the sub-index $j$ represents the $j$-th component of the vector $\Delta \boldsymbol{q}$, we collect all these constraints in the linear equation system written in terms of the vector $\Delta \boldsymbol{q}$ :

$$
\boldsymbol{I}^{c} \Delta \boldsymbol{q}=\mathbf{0}
$$

where $\boldsymbol{I}^{c} \in \mathbb{R}^{n_{c} \times n}$ is a matrix with ones in the positions $(k, j)\left(I_{k j}^{c}=1\right)$ and zeros in the remaining positions. The index $k\left(k=1, \ldots, n_{c}\right)$ corresponds to the $k$ equation, and this equation prescribes to zero the $j$-th d.o.f. of the vector $\Delta \boldsymbol{q}$.

Using this notation, the $\tilde{\mathscr{U}}_{q}^{\text {Lin }}$ space, defined in equation (47), that represents the Linear boundary displacement micro-cell sub-model, is rewritten as:

$$
\tilde{\mathscr{U}}_{q}^{\text {Lin }}=\left\{\Delta \boldsymbol{q} \mid \boldsymbol{I}^{c} \Delta \boldsymbol{q}=\boldsymbol{0}\right\}
$$

where it is understood that there are as many equations as d.o.f.'s prescribed to zero in the boundary $\Gamma_{\mu}$.

Case ii) Rigid link imposing identical displacement fluctuations between a node and a point (Figure 5): : classical periodic boundary condition.

Let us consider a rigid connection linking the displacement fluctuation of a node $k \in \Gamma_{\mu}^{+}$, in the position $\boldsymbol{y}^{+}$, and the displacement fluctuation of the point $\boldsymbol{y}^{-}$:

$$
\Delta \boldsymbol{q}_{k}=\Delta \tilde{\boldsymbol{u}}_{\mu}\left(\boldsymbol{y}^{-}\right)=\sum_{i=1}^{n_{\text {node }}^{\mu}} N_{i \mu}\left(\boldsymbol{y}^{-}\right) \Delta \boldsymbol{q}_{i}
$$

the points $\left(\boldsymbol{y}^{+}, \boldsymbol{y}^{-}\right)$form a pair as explained in the item $(c)$ above. Note that the right hand term is the nodal value of the displacement fluctuation increment interpolated in the point $\boldsymbol{y}^{-}$.

In a Periodic sub-model, as shown in Figure 5, $n_{p}$ vectorial equations like (52) have to be prescribed, with $n_{p}$ the number of nodes in $\Gamma_{\mu}^{+}$. Then, the total system of equations can be written as follows:

$$
\left(I^{p}-N_{\mu}\right) \Delta \boldsymbol{q}=\mathbf{0}
$$

where the matrix $\boldsymbol{I}^{p} \in \mathbb{R}^{\left(2 n_{p} \times n\right)}$ is a matrix with ones in the positions $(k, l)\left(\boldsymbol{I}_{k l}^{p}=1\right)$ and zeros in the remaining positions. The index $k$ corresponds to the $k$-th equation, and this equation imposes the link restriction of the $l$-th d.o.f. in $\Delta \boldsymbol{q}$. The point $\boldsymbol{y}^{-} \in \Gamma_{\mu}^{-}$, which displacement fluctuation is prescribed through equation $k$-th, is associated with the shape functions $N_{i \mu}\left(y^{-}\right)$. These shape functions are used to assemble the Matrix $\boldsymbol{N}_{\mu} \in \mathbb{R}^{\left(2 n_{p} \times n\right)}$ given in expression (53).

With this notation, the spaces $\tilde{\mathscr{U}}_{q}^{\text {Per }}$ can be rewritten as follows:

$$
\tilde{\mathscr{U}}_{q}^{\text {Per }}=\left\{\Delta \boldsymbol{q} \quad \mid \quad\left(\boldsymbol{I}^{p}-\boldsymbol{N}_{\mu}\right) \Delta \boldsymbol{q}=\boldsymbol{0}\right\}
$$

Periodic kinematical constraints, imposed on the complete boundary $\Gamma_{\mu}=\Gamma_{\mu}^{-} \cup \Gamma_{\mu}^{+}$, preclude rigid body rotations but not rigid body translations. Thus, in order to define a mechanically well-posed discrete variational problem, two additional d.o.f.'s should be prescribed using equation (50), as sketched in Figure 5. 


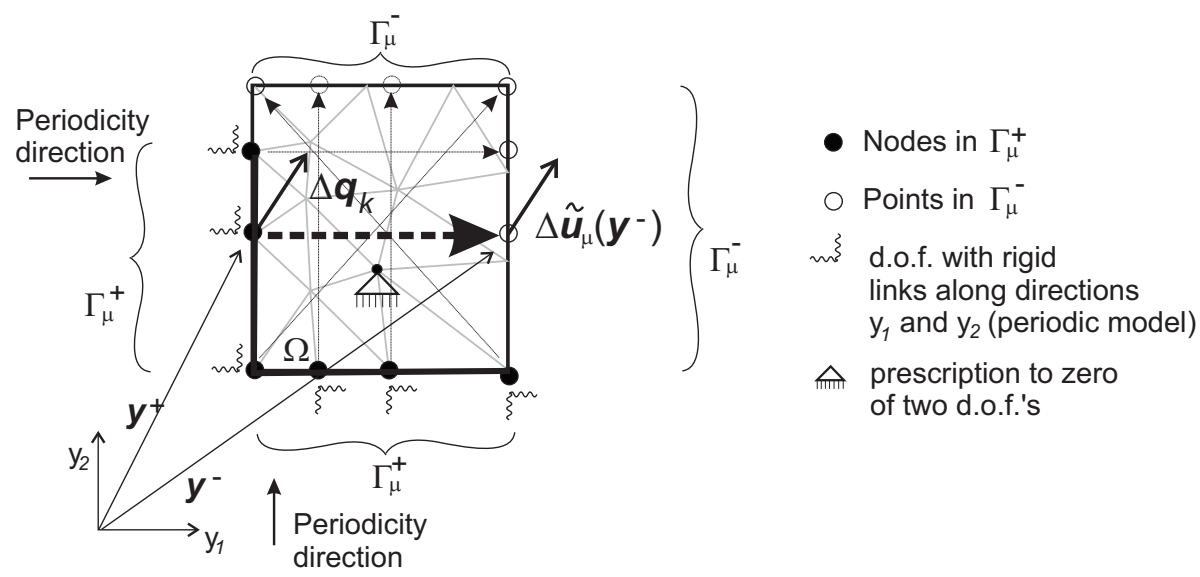

Figure 5: Micro-cell $\Omega_{\mu}$ and finite element mesh with classical periodic (rigid links) boundary conditions.

Furthermore, when using the periodic condition (52) in the boundary, and particularly when $\boldsymbol{y}^{-}$does not coincides with a finite element mesh nodal point, we have observed that a good practice is to introduce one additional kinematical constraint similar to the one presented in the following item case iii in order to improve the accuracy.

Case iii) Imposition of a null volumetric average of strain fluctuation increments in a region $\breve{\Omega}$ bounded by the surface $\breve{\Gamma}$ (Figure 6).

Equation (8) prescribes the minimum kinematical constraint in $\Omega_{\mu}$, meaning that the volumetric average in $\Omega_{\mu}$ of the strain fluctuation increments are null. A similar kinematical constraint can be prescribed in domains $\breve{\Omega} \subseteq \Omega_{\mu}$ bounded by closed surfaces $\breve{\Gamma}$ and with normal vectors $\breve{v}$. This kind of prescriptions on $\breve{\Gamma}$, including the specific case when $\breve{\Gamma} \equiv \Gamma_{\mu}$, can be rewritten as follows:

$$
\boldsymbol{H} \Delta \boldsymbol{q}=\mathbf{0}
$$

where the matrix $\boldsymbol{H} \in \mathbb{R}^{3 \times n}$ is the assembling of "nel" finite element matrices $\boldsymbol{H}^{e}$ (nel is the number of finite elements in the mesh belonging to the domain $\breve{\Omega}$, bounded by $\breve{\Gamma}$, and having at least one side in $\breve{\Gamma}$ ):

$$
\boldsymbol{H}=\bigwedge_{e=1}^{\mathrm{nel}} \int_{\breve{\Gamma}^{e}} \boldsymbol{H}^{e} d \breve{\Gamma}^{e}
$$

with $\breve{\Gamma}^{e}$ being the finite element side that belongs to the boundary $\breve{\Gamma}$. The matrix $\boldsymbol{H}^{e}$ is given by:

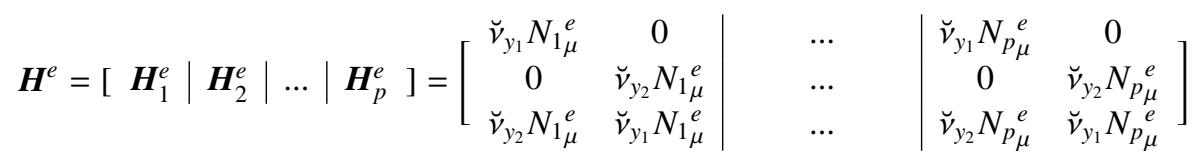

that is formed by $p$ ( $p$ is the number of finite element nodes) matrix blocks of dimension: $\mathbb{R}^{3 \times 2}$, one of this block for every finite element node. Implicit in equations (56) and (57) is the concept that the matrix block $\boldsymbol{H}_{l}^{e}\left(\mathbb{R}^{3 \times 2}\right)$ associated with the node $l$ not staying on the boundary $\breve{\Gamma}$, is null.

From these expressions, the space $\tilde{\mathscr{U}}_{q}^{\text {Min }}$ can be defined by means of the following condition:

$$
\tilde{\mathscr{U}}_{q}^{\text {Min }}=\{\Delta \boldsymbol{q} \mid \boldsymbol{H} \Delta \boldsymbol{q}=\boldsymbol{0}\}
$$

where the integration boundary, $\breve{\Gamma}$, is chosen according to the RVE sub-model.

The kinematical constraints given by equation (55), involving the complete external boundary $\Gamma_{\mu}$, does not preclude micro-cell rigid body motions. Thus, three additional d.o.f. constraints (50) must be added to the sub-model in order to define a well-posed incremental variational problem, as it is sketched in Figure 6.

Case iv) Hybrid constraints: minimum kinematical constraints with imposition of rigid links on boundary nodes along arbitrary directions (Figure 7). The motivation of using this kind of boundary conditions on $\Gamma_{\mu}$ is discussed in Section 4. 


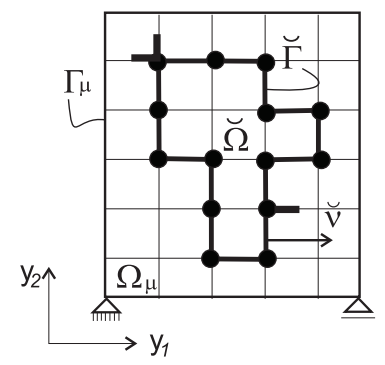

- Nodes in $\breve{\Gamma}$

- Horizontal d.o.f.'s, minimum kinematical constraint

| Vertical d.o.f.'s, minimum kinematical constraint

Aाm Prescription to zero of two d.o.f.'s

$\triangle$ Prescription to zero of one d.o.f.'s

Figure 6: Micro-cell $\Omega_{\mu}$ and finite element mesh with a null average value of the strain fluctuation increments in the region $\breve{\Omega}$ bounded by the curve $\breve{\Gamma}$.

Let us define rigid connections, or links, prescribing identical displacement fluctuation increments between boundary nodes lying along an arbitrary direction, not necessarily parallel to the micro-cell sides. The pair of points $\boldsymbol{y}^{+}$(in $\Gamma_{\mu}^{+}$) and $\boldsymbol{y}^{-}$(in $\Gamma_{\mu}^{-}$), which are constrained to have identical displacement fluctuation increments, are the intersection points between the segment parallel to the given direction (called periodicity direction) with the boundary $\Gamma_{\mu}$ of the RVE, as shown in Figure 7 . The prescriptions are imposed on $\boldsymbol{y}^{+}$and $\boldsymbol{y}^{-}$by means of equations similar to (52). This constraint is called the generalized periodic boundary condition.

In this general case, even when identical displacements are imposed on boundary points, because the normal vectors do not necessarily satisfy the identity: $\boldsymbol{v}_{\mu}^{-}=-\boldsymbol{v}_{\mu}^{+}$, then, the constraint (8) is not automatically guaranteed and it has to be explicitly imposed. Otherwise, the volumetric average strain fluctuation increments shall not be necessarily zero.

Furthermore, any rigid body translation should be explicitly precluded by adding two d.o.f. constraints of the type given by equation (50).

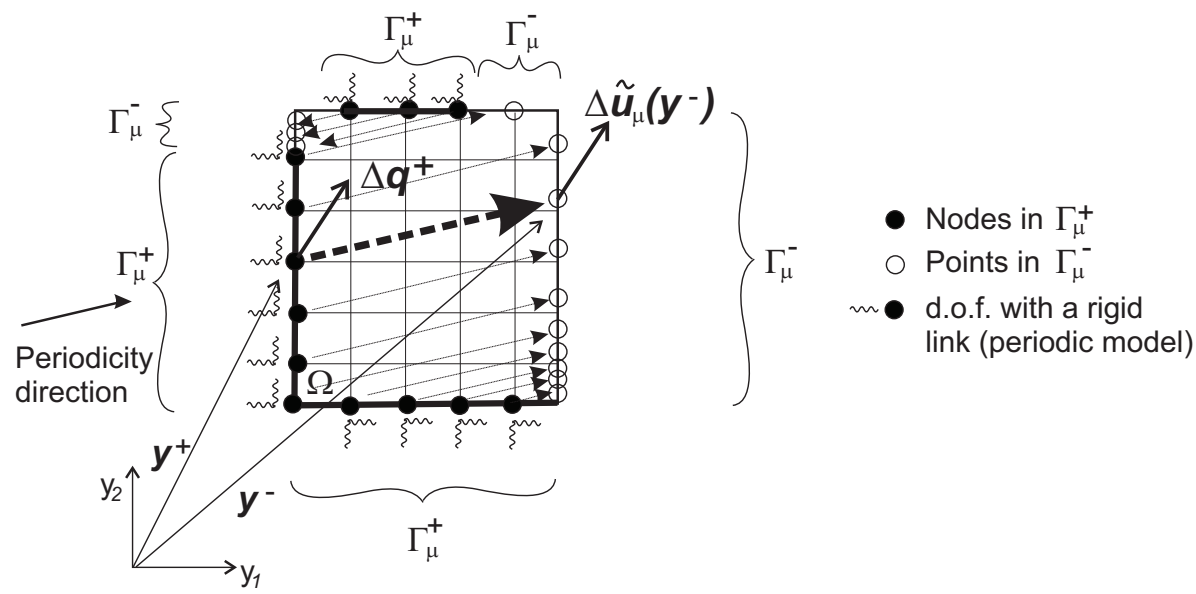

Figure 7: Micro-cell $\Omega_{\mu}$ and finite element mesh. Hybrid boundary condition: periodicity along arbitrary directions which satisfies a null strain fluctuation increments in the region bounded by the curve $\Gamma_{\mu}$.

All these kinds of kinematical constraints are imposed through the following blocks of the linear system of equations:

$$
\begin{aligned}
\boldsymbol{I}^{c} \Delta \boldsymbol{q} & =\boldsymbol{0} ; \\
\left(\boldsymbol{I}^{p}-\boldsymbol{N}_{\mu}\right) \Delta \boldsymbol{q} & =\boldsymbol{0} ; \\
\boldsymbol{H} \Delta \boldsymbol{q} & =\boldsymbol{0} .
\end{aligned}
$$

Thus, there are $n_{c}$ equations of type (59), $2 n_{p}$ equations of type (60) and three equations of type (61) for the RVE boundary $\Gamma_{\mu}$.

Additional details about the numerical implementation of the kinematical constraints described in the above cases i) to iv) are given in Appendix A. 


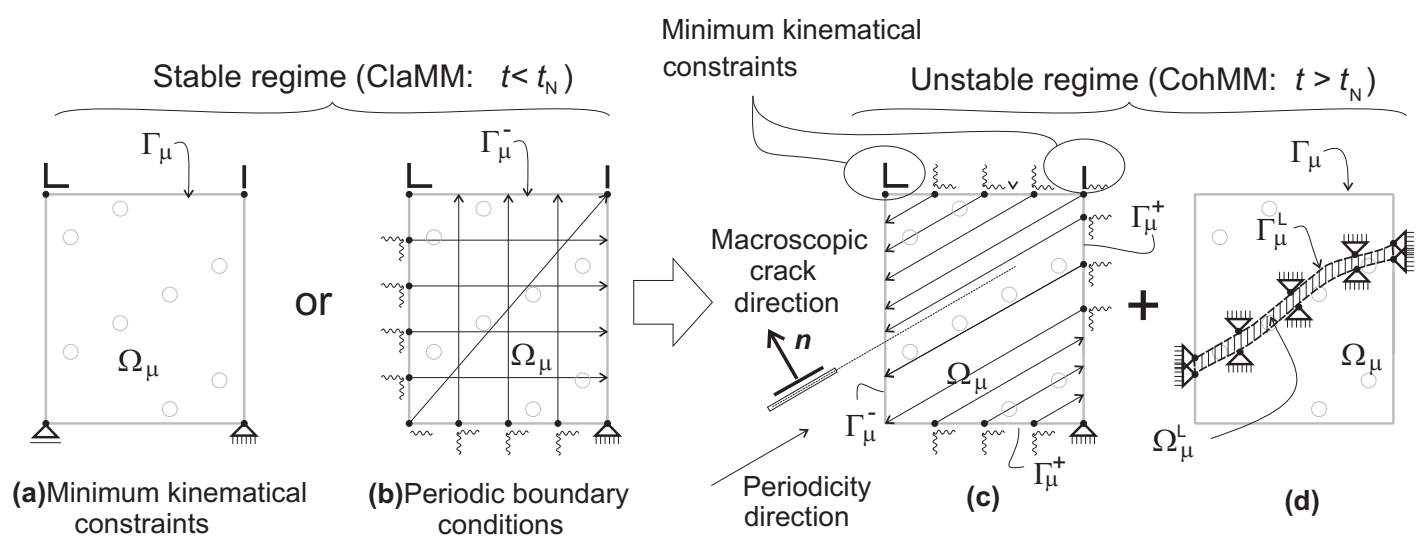

Figure 8: Micro-cell sub-models for the analysis of multiscale fracture problems. Boundary conditions on the displacement fluctuation field: the symbols agree with those defined in Figure 5. During the macroscopic stable regime, the standard boundary conditions (SBC) are prescribed. After bifurcation $\left(t \geq t_{N}\right)$, generalized periodic conditions are conveniently imposed, aligned with the crack direction (c), and new boundary conditions (NSBC) are introduced (d).

\section{Technique to perform the RVE failure analysis}

As mentioned above, the RVE standard boundary conditions (SBC) define the ClaMM sub-model adopted for the analysis during the stable response of the material. Minimum kinematical constraint or periodic sub-models, such as sketched in Figures 8-a and 8-b, can typically be used in this stage of the analysis.

After the bifurcation time $\left(t \geq t_{N}\right)$, the ClaMM model is changed by the CohMM model. To perform this change, the localization domain $\Omega_{\mu}^{L}$, sketched in Figure 8-d, must be clearly identified. Then, the nonstandard boundary conditions (NSBC) are prescribed along the boundary $\Gamma_{\mu}^{L}$ and the strain rates: $\left(d \boldsymbol{\beta} \otimes^{s}\right.$ $\left.\boldsymbol{n}_{\mu}\right) / \ell_{\mu}$ are injected into $\Omega_{\mu}^{L}$, according to equation (27).

A technique which allows to determine $\Omega_{\mu}^{L}$ and introduces the NSBC, as well as allows to induce the complete degradation of the homogenized material response, consists of two sequential steps which are described as follows:

i) First step, immediately after $t_{N}$ is detected:

Regardless of the RVE sub-model defined in the stable regime, at $t=t_{N}$ we change the SBC to generalized periodic boundary conditions on $\Gamma_{\mu}$ having the periodicity direction orthogonal to $n$, or equivalently, parallel to the discontinuity surface introduced at the macroscopic scale. This technique follows the ideas proposed in the work of Coenen et al. [6], where similar kinematical constraints are called the percolation-path-aligned boundary conditions. The so-considered periodic boundary conditions are sketched in Figure 8-c, and they are prescribed in the model by adding the corresponding set of equations (53).

In addition to these periodic boundary conditions aligned with the macro-crack, we also enforce the minimum kinematical constraints on $\Gamma_{\mu}$ by adding a complementary set of three equations of the type (55). In Figure 8-c, we depict a possible selection of dependent d.o.f.'s associated to these equations.

The introduction of the periodic boundary conditions aligned with the macro-crack intensifies the strain localization zone evolution. This behavior is induced by the microscopic failure processes after bifurcation, as well as allows for the elastic unloading of the neighbor zones evidencing more clearly the detection of $\Omega_{\mu}^{L}$. Thus, introducing the periodic boundary conditions aligned with the macrocrack into the model, and after a short period of time, $\Omega_{\mu}^{L}$ and its boundary $\Gamma_{\mu}^{L}$ can be identified using the following criteria: $\boldsymbol{y} \in \Omega_{\mu}^{L}$, iff, $\boldsymbol{n} \cdot d \tilde{\boldsymbol{\varepsilon}}_{\mu}(\boldsymbol{y}) \cdot \dot{\boldsymbol{\beta}}>0$, where $d \tilde{\boldsymbol{\varepsilon}}_{\mu}(\boldsymbol{y})$ is the micro strain fluctuation increments defined in equation (7).

ii) Second step, a short time after the periodic boundary conditions aligned with the macro-crack have been prescribed and the domain $\Omega_{\mu}^{L}$ has been determined, the NSBC are introduced in the model. From the wide class of NSBC that can be imposed on $\Gamma_{\mu}^{L}$, we adopt the criterion of defining a Taylor sub-model or linear displacement boundary conditions. Both of them allow for managing the cases where $\Omega_{\mu}^{L}$ intersects internal voids, as shown in Figure 8-d. 


\section{Numerical Model Assessment}

In this Section, three numerical examples are presented in order to assess the multiscale failure model presented in the previous sections.

The first one, described in Sub-Section 5.1, is a detailed failure analysis of several micro-cell submodels constructed with different boundary conditions. The example is addressed to evaluate the capability that these sub-models have to capture the failure mode and the associated homogenized tractions for one macroscopic problem having a heterogeneous microstructure. In addition, this example allows us to represent the evolution of the postcritical loading process until reaching the complete degradation of the RVE, which is represented by a null homogenized traction vector for a given displacement jump.

The second example, presented in Sub-Section 5.2, shows a two-scale analysis of a structural problem. We solve the structure by using Direct Numerical Simulation (DNS) and the solutions are then compared to the numerical results supplied by the FOMF approach.

In the last example presented in Sub-Section 5.3, we simulate the failure of a strip undergoing uniaxial tensile. This numerical test shows the capability of the multiscale model to simulate failure mechanisms governed by micro-cracks displaying zigzag, or arbitrary, paths.

\subsection{Fracture process analysis of a heterogeneous material point.}

\subsubsection{Microstructure description.}

The case of study corresponds to a material having a microstructure with a periodic distribution of microvoids, such as shown in Figure 9-a. The microvoid periodicity line, along which the distance between microvoids is closer, forms an angle $\alpha=30$. [deg] with the horizontal direction. The remaining length defining the microstructure is the minimum distance $h$ between voids. Also, Figure 9-a shows a unit cell with height $4 h$ denoted Cell $_{1 \times 1}$.

The base material in the micro-structure is characterized with a damage model with an exponential softening (for a detailed description of this material model see [30] and [20]). The model parameters are: Young's modulus: $E=30$ [GPa], Poisson's ratio: $v=0.3$, ultimate stress: $f_{t}=2.5[\mathrm{MPa}]$ and fracture energy: $G_{f}=10 \mathrm{~N} / \mathrm{m}$. The numerical simulations of the micro-cells are performed by using a smeared crack approach. Then, the softening modulus of the continuum damage model is regularized with the fracture energy and the finite element size according to the technique described in [30].

Notice that any point of the microstructure can undergo damage and strain localization. As well as, there are not strain localization bands predefined in the microstructure.

\subsubsection{Loading condition of the micro-cells.}

Initially, before detecting the macrosocopic bifurcation, the macroscopic point represented by the unit cell, Cell ${ }_{1 \times 1}$, is undergoing an uniaxial vertical stretching process being modeled with the ClaMM approach. In this stage, the macroscopic strain is given by $\boldsymbol{\varepsilon}_{R}=\left[\boldsymbol{\varepsilon}_{R x_{1}}, \boldsymbol{\varepsilon}_{R x_{2}}, \boldsymbol{\varepsilon}_{R x_{1} x_{2}}\right]=\left[0, f_{1}(t), 0\right]$, where $f_{1}(t)$ is a linear increasing function in time, as depicted in Figure 9-b.

Once the macroscopic bifurcation time is detected $\left(t=t_{N}\right)$, the unit cell model is changed according with the CohMM. We assume that the macroscopic strain, that is compatible with a strain localization mode, is given by: $\varepsilon=\varepsilon_{R}+\varepsilon_{D}$, where $\varepsilon_{R}(t)$ corresponds to the regular part of the strain and is defined identically as in the previous paragraph, with the function $f_{1}(t)$ now decreasing to zero, while $\boldsymbol{\varepsilon}_{D}=\left(\boldsymbol{n} \otimes^{s} \boldsymbol{\beta}\right) / \ell_{\mu}$ typifies the term related to the displacement jump in the macroscopic band. In this example, we define $\beta(t)=f_{2}(t) \ell_{\mu} \gamma$, with $f_{2}(t)$ being a monotonous increasing function of time plotted in Figure 9-b. The vectors $\boldsymbol{n}$ and $\boldsymbol{\gamma}$ are the eigenvectors of the acoustic tensor which are determined at the bifurcation time. The vector $\boldsymbol{n}$ represents the normal vector to the the discontinuity surface at the macroscale. And, as can be observed in the following numerical results, it is almost identical to the unit vector $\boldsymbol{n}_{\mu}$, that is orthogonal to the strain localization band at the microscopic scale.

The macroscopic strain increment $\varepsilon$, is injected into the micro-cell as dictated by the CohMM approach, i.e., $\boldsymbol{\varepsilon}_{R}(t)$ is uniformly distributed in the unit cell, while $\boldsymbol{\varepsilon}_{D}$ is uniformly distributed in $\Omega_{\mu}^{L}$. 


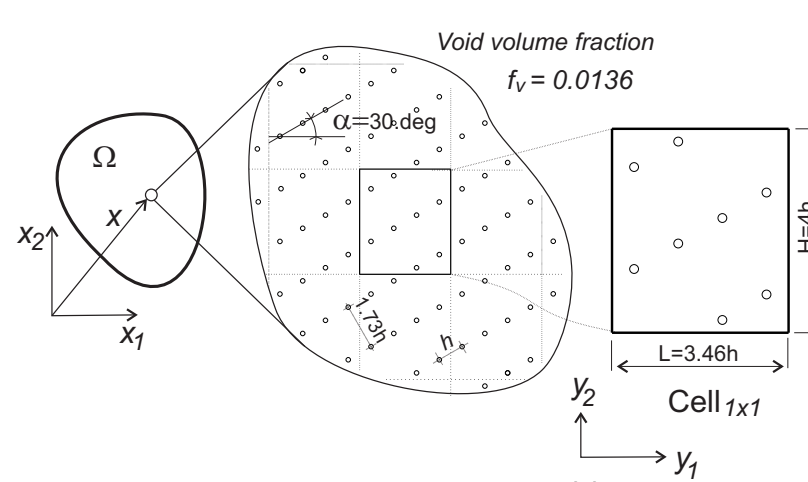

(a)

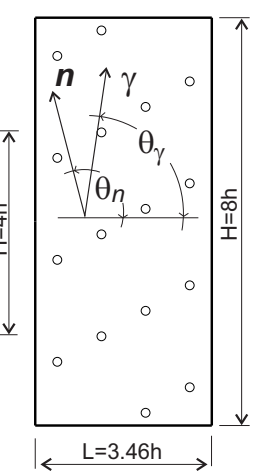

Cell $1 \times 2$

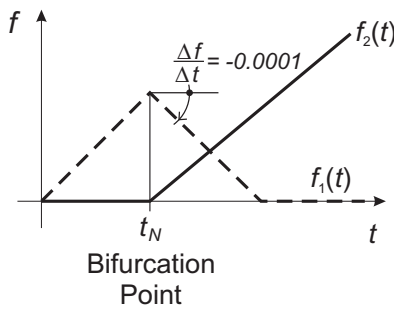

(b)

Figure 9: Fracture process analysis of a heterogeneous material point. a) Material microstructure and Cell definitions, b) pseudo-time dependent curves defining the evolution of the macrostrains.

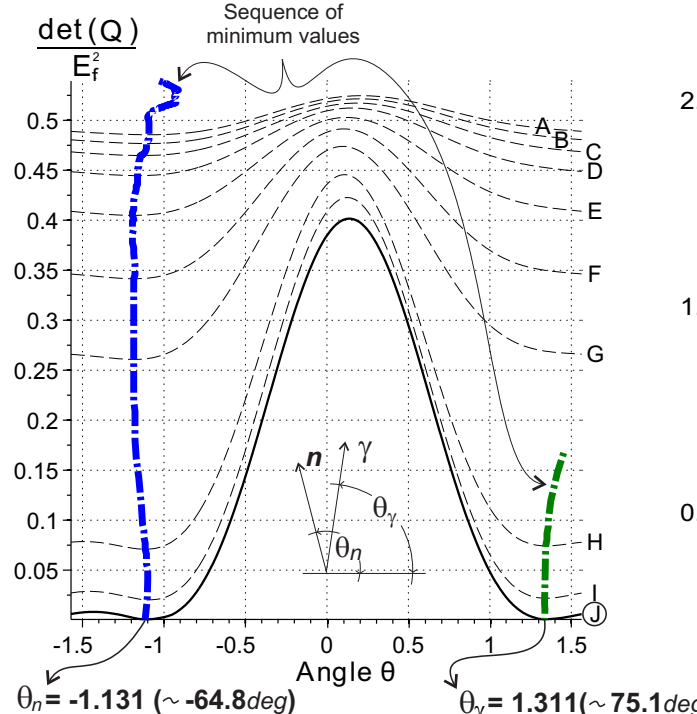

(a)

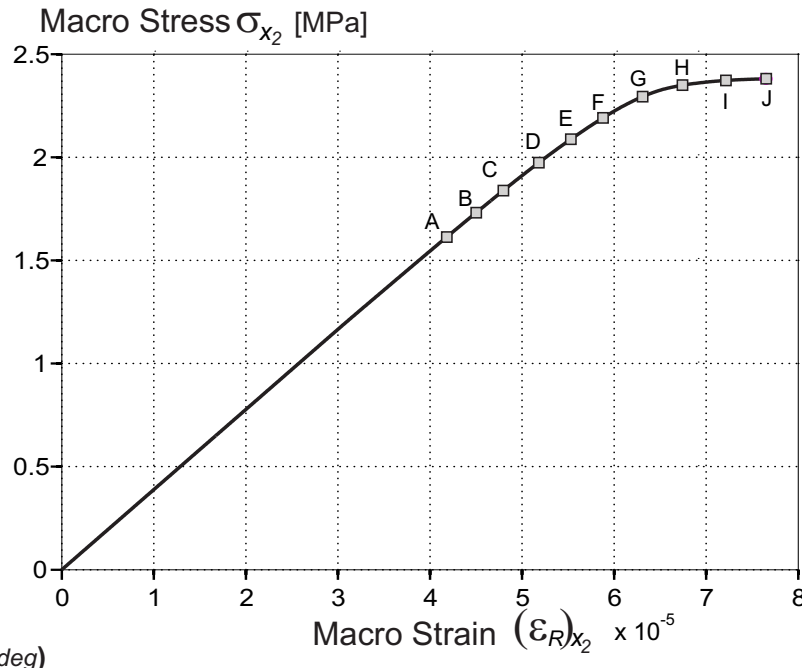

(b)

Figure 10: Analysis of the strain localization mode. a) Evolution of $\operatorname{det}(\boldsymbol{Q}(\boldsymbol{n}(\theta), t))$ for the Cell ${ }_{1 \times 1}$ with periodic boundary conditions. b) homogenized macro stress vs. macro strain. Letters identify the time when $\operatorname{det}(\boldsymbol{Q})$ has been evaluated.

\subsubsection{Analysis of the strain localization mode detected with different cell models.}

Since the microstructure is periodic, the unit cell Cell ${ }_{1 \times 1}$ with periodic boundary conditions satisfies the criterion for constituting a RVE, at least for the macroscopic stable material regimen. However, the Cell $1_{1 \times 1}$ with minimum kinematical constraint, or with linear displacements on the boundary, does not satisfy that criterion, i.e., they are not RVE.

First, we analyze the strain localization mode obtained with the ClaMM and $\mathrm{Cell}_{1 \mathrm{x} 1}$ with periodic boundary conditions. The strain localization mode is typified by the angles $\theta_{\boldsymbol{n}}$ and $\theta_{\gamma}$, the time of bifurcation $t_{N}$ and the homogenized stress levels at $t_{N}$. The solution is taken as the exact one and is compared with the same variables obtained using cells with increasing sizes and subjected to minimally constrained or linear boundary displacements in order to estimate the required cell size providing similar solutions to the unit cell with periodic boundary conditions.

The loss of macroscopic material stability is tested during the simulation. Figure 10 shows the evolution of $\operatorname{det}(\boldsymbol{Q}(\boldsymbol{n}(\theta), t))$ vs. the angle $\theta$ formed by the vector $\boldsymbol{n}$ and the horizontal direction in different stages of the analysis. The determinant is normalized with the square of an effective Young's modulus: $E_{f}=\left(1-f_{v}\right) E$, being $f_{v}$ the microvoid volume fraction and $E$ is the Young's modulus of the bulk material. These results have been obtained with the Cell ${ }_{1 \times 1}$. Also, in this Figure, we plot the evolution of the two minimum values of $\operatorname{det}(\boldsymbol{Q})$. The bifurcation time corresponds to the instant when these curves intersect the horizontal axis (Point $\mathrm{J}$ ). Additionally, the two angles given by the minimum values of the determinant correspond with the two eigenvectors of the acoustic tensor: $\boldsymbol{n}$ and $\boldsymbol{\gamma}$ and their angles are denoted $\theta_{\boldsymbol{n}}$ and $\theta_{\gamma}$, respectively. 


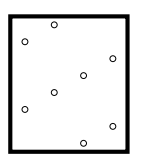

Cell $_{1 \times 1}$

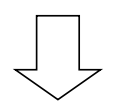

2290 elements

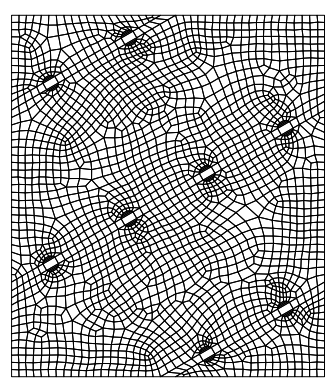

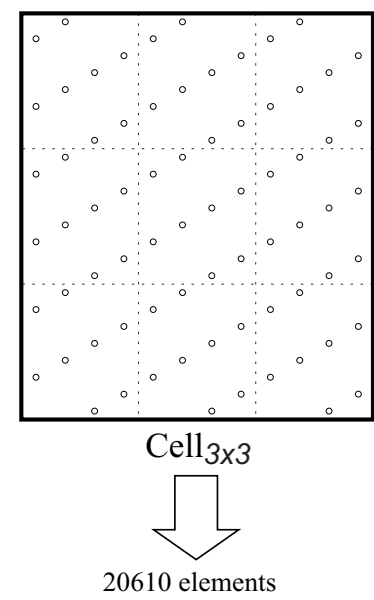

20610 elements

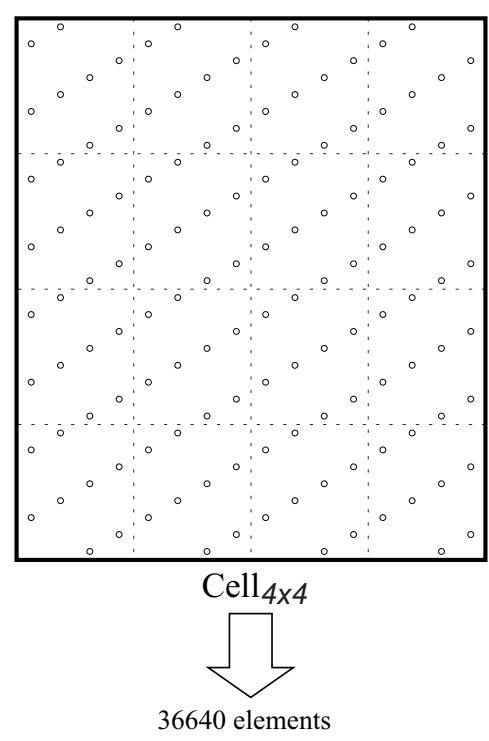

36640 elements

Figure 11: A sequence of cells taken to analyze the localization modes using minimally constrained or linear boundary displacements.

\begin{tabular}{|c|c|c|c|c|c|c|}
\hline sub-model & Cell size & $t_{N}$ & $\sigma_{x_{2}}^{N}[\mathrm{MPa}]$ & $\left(\varepsilon_{R}\right)_{x_{2}}^{N}$ & $\theta_{\boldsymbol{n}}[\mathrm{deg}]$ & $\theta_{\gamma}[\mathrm{deg}]$ \\
\hline \hline \multirow{4}{*}{$\begin{array}{c}\text { Periodic } \\
\text { boundary conditions }\end{array}$} & Cell $_{1 \times 1}$ & 0.766 & 2,380 & $7.65 \mathrm{e}-5$ & -64.8 & 75.1 \\
\cline { 2 - 7 } & $\mathrm{Cell}_{2 \times 2}$ & 0.766 & 2,380 & $7.65 \mathrm{e}-5$ & -64.8 & 75.1 \\
\cline { 2 - 7 } & $\mathrm{Cell}_{3 \times 3}$ & 0.766 & 2,380 & $7.65 \mathrm{e}-5$ & -64.8 & 75.1 \\
\hline \hline \multirow{3}{*}{$\begin{array}{c}\text { Minimum kinematical } \\
\text { boundary conditions }\end{array}$} & $\mathrm{Cell}_{1 \times 1}$ & 0.694 & 2,346 & $6.93 \mathrm{e}-5$ & -70.2 & 75.6 \\
\cline { 2 - 7 } & $\mathrm{Cell}_{2 \times 2}$ & 0.694 & 2,354 & $6.93 \mathrm{e}-5$ & -69.3 & 76.0 \\
\cline { 2 - 7 } & $\mathrm{Cell}_{3 \times 3}$ & 0.704 & 2.359 & $7.03 \mathrm{e}-5$ & -68.0 & 75.6 \\
\cline { 2 - 7 } & $\mathrm{Cell}_{4 \times 4}$ & 0.705 & 2.361 & $7.04 \mathrm{e}-5$ & -67.9 & 76.0 \\
\hline \hline \multirow{3}{*}{$\begin{array}{c}\text { Linear displacement } \\
\text { boundary conditions }\end{array}$} & $\mathrm{Cell}_{1 \times 1}$ & 0.890 & 2.492 & $8.99 \mathrm{e}-5$ & -68.4 & 81.0 \\
\cline { 2 - 7 } & $\mathrm{Cell}_{2 \times 2}$ & 0.801 & 2.425 & $8.00 \mathrm{e}-5$ & -65.2 & 77.4 \\
\cline { 2 - 7 } & $\mathrm{Cell}_{3 \times 3}$ & 0.770 & 2.405 & $7.69 \mathrm{e}-5$ & -64.8 & 76.0 \\
\cline { 2 - 7 } & $\mathrm{Cell}_{4 \times 4}$ & 0.757 & 2.396 & $7.56 \mathrm{e}-5$ & -64.8 & 75.6 \\
\hline
\end{tabular}

Table 1: Capture of strain localization modes using different cell sizes and sub-models.

Note that, under the loading condition studied in this example, the angle $\theta_{\boldsymbol{n}}$ defines a vector $\boldsymbol{n}$ which is almost orthogonal to the line along which the distance between micro-voids are closer, characterizing a macro crack which is parallel to that direction.

Next, we analyze the localization modes determined with a sequence of cells, such as that depicted in Figure 11. They are denoted $\mathrm{Cell}_{1 \times 1}, \mathrm{Cell}_{2 \times 2}, \mathrm{Cell}_{3 \times 3}, \mathrm{Cell}_{4 \times 4}$, and are obtained by the horizontal and vertical repetition of the $\mathrm{Cell}_{1 \mathrm{x} 1}$. Those cells are subjected to minimally constrained or linear boundary displacements.

The bifurcation mode solutions obtained with the four sequence of cells are presented in Table 1, where the following variables are depicted: $i$ ) the bifurcation time $t_{N}$, ii) both angles, $\theta_{\boldsymbol{n}}$ and $\theta_{\gamma}$ and iii) the homogenized stress $\sigma_{x_{2}}$ and strain $\left(\varepsilon_{R}\right)_{x_{2}}$ levels at $t_{N}$.

Figure 12-a shows a zoom, close to $t_{N}$, of the $\sigma_{x_{2}}$ vs. $\left(\varepsilon_{R}\right)_{x_{2}}$ solution curves that have been obtained with the sequence of four cells using the linear displacement boundary condition sub-model. These curves are compared with the solution obtained using periodic boundary conditions. Also, Figure 12-b plots the logarithmic error of the homogenized stress at the bifurcation time $t_{N}$ vs. the logarithmic cell sizes. The (relative) error is defined as:

$$
e=\frac{\left\|\sigma_{N}^{*}-\sigma_{N}^{p}\right\|}{\left\|\sigma_{N}^{p}\right\|}
$$

where $\sigma_{N}^{*}$ denotes the stress $\sigma_{x_{2}}$ at the bifurcation time $t_{N}$ evaluated with the minimum kinematical constraint or linear boundary displacement sub-models, and $\sigma_{N}^{p}$ denotes the same stress determined with the periodic boundary condition sub-model.

As can be observed from Table 1 and Figure 12, in all cases, the sequence of the stress level reached at $t_{N}$ tends to the value furnished by the periodic solution. The linear boundary displacement sub-models show a higher convergence rate, see Figure 12-b. The angles $\theta_{\boldsymbol{n}}$ and $\theta_{\gamma}$, and $t_{N}$ show a correct trend only in those cases corresponding to the linear boundary displacement sub-models. However, the solutions provided by 


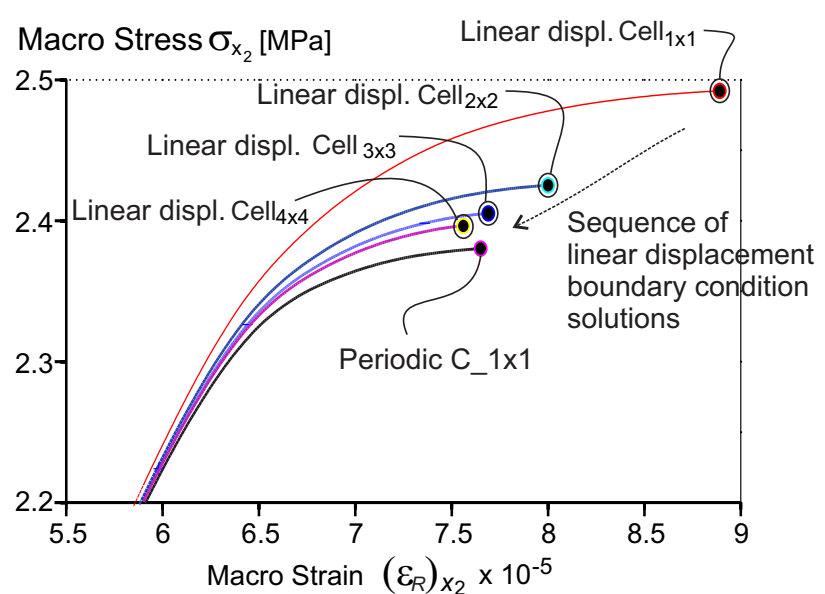

(a)

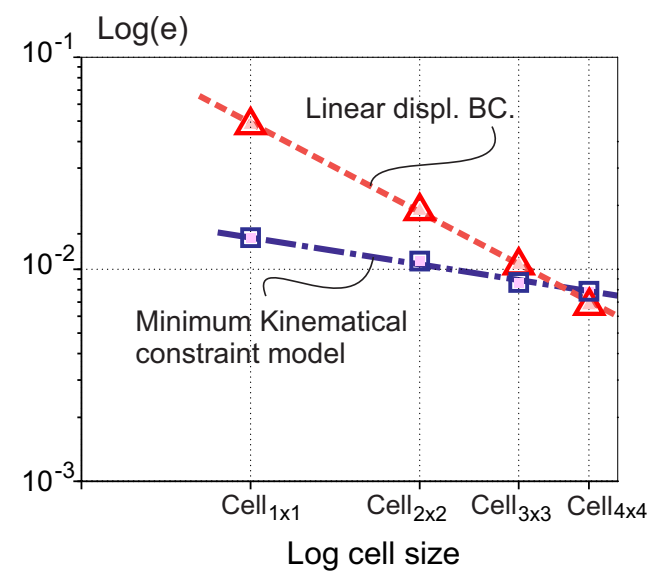

(b)

Figure 12: a) Curves $\sigma_{x_{2}}$ vs. $\boldsymbol{\varepsilon}_{x_{2}}$ of the linear displacement boundary condition sub-model solutions compared with the Cell ${ }_{1 \times 1}$ and periodic conditions, the end of the curves represent the bifurcation point at $t_{N}$; b) error curves of the bifurcation stresses for different sub-models vs. cell sizes.

the sequence of minimum kinematical constraint models display limit values which are slightly different. We can explain this inconsistency by the fact that, when we use minimum kinematical constraint models, the micro cells have to be slightly modified in order to avoid the very marked bias induced by voids which are close to the boundaries with uniform tractions. Thus, we have decided to remove the voids, which are close to the boundaries, from the cells.

With this analysis we get an estimation of the cell size satisfying the condition which are required to be considered a RVE. A similar analysis to determine the RVE size by means of a convergence analysis has been performed by Pindera et al. [36]. They have also used several cells with linear displacement and minimum kinematical boundary condition sub-models. However, the Pindera's et al. study has been restricted only to elastic heterogeneous materials with periodic microstructure, while in the present case we are extending the discussion by incorporating failure mechanisms in the analysis.

\subsubsection{Analysis of objectivity of the CohMM results in the postcritical regime.}

After detecting the bifurcation condition, the generalized macro-kinematics is injected are injected in the cell as dictated by the CohMM approach, and boundary conditions are modified consequently. In this case, we select a linear displacement boundary condition that is prescribed on $\Gamma_{\mu}^{L}$, while, in $\Gamma_{\mu}$ we adopt boundary conditions with a periodicity direction orthogonal to $\boldsymbol{n}$.

In order to analyze the objectivity of results with respect to the cell size, we adopt two cells denoted Cell $_{1 \times 1}$ and Cell ${ }_{1 \times 2}$, as shown in Figure 9.

Figure 13 shows the finite element meshes employed for modeling both cells: Cell $_{1 \times 1}$ and Cell $1_{1 \times 2}$ and the localization domain $\Omega_{\mu}^{L}$ evaluated at $t_{N}$ in each case. Note that, in order to include parts of the voids into $\Omega_{\mu}^{L}$, the voids are discretized with the finite element mesh and they are modeled by assuming an elastic material with a very small Young's modulus.

Figure 14 displays the damage map and deformed meshes, defined by the displacement fluctuation field, of both cells and at two different stages: at the bifurcation time $t_{N}$ and at the end of the analysis. The damage level obtained in the pre-bifurcation stage is notably high. We remark the fact that damage evolution, during the pre-bifurcation stage, determines a non-linear homogenized macroscopic constitutive response displaying, at this level of analysis, a stable energetic dissipation.

The normal and tangential components of the homogenized traction vector $\boldsymbol{T}$ as a function of the macroscopic displacement jump $\boldsymbol{\beta}$ are plotted in Figure 15. The vector $\boldsymbol{T}$ is computed during the post-critical macroscopic regime by using the CohMM approach. Solutions obtained with the $\mathrm{Cell}_{1 \times 1}$ and $\mathrm{Cell}_{1 \times 2}$ are included in the plots. Note that solutions supplied by both cells are almost identical, verifying the objectivity in the material response

Also, the pseudo-time continuity of $\boldsymbol{T}$, at the bifurcation time when changing from the ClaMM to the CohMM model, has been determined. Defining the traction vector $\boldsymbol{T}_{C l a M M}=\boldsymbol{\sigma} \boldsymbol{n}$ with $\boldsymbol{\sigma}$ being the homogenized stress obtained with the ClaMM approach and with $\boldsymbol{T}_{C o h M M}$ the homogenized traction vector obtained with the CohMM approach, and a relative error norm as: $e_{T}=\left(\left\|\boldsymbol{T}_{C o h M M}-\boldsymbol{T}_{\text {ClaM }}\right\|\right) /\left\|\boldsymbol{T}_{\text {CohMM }}\right\|$; 


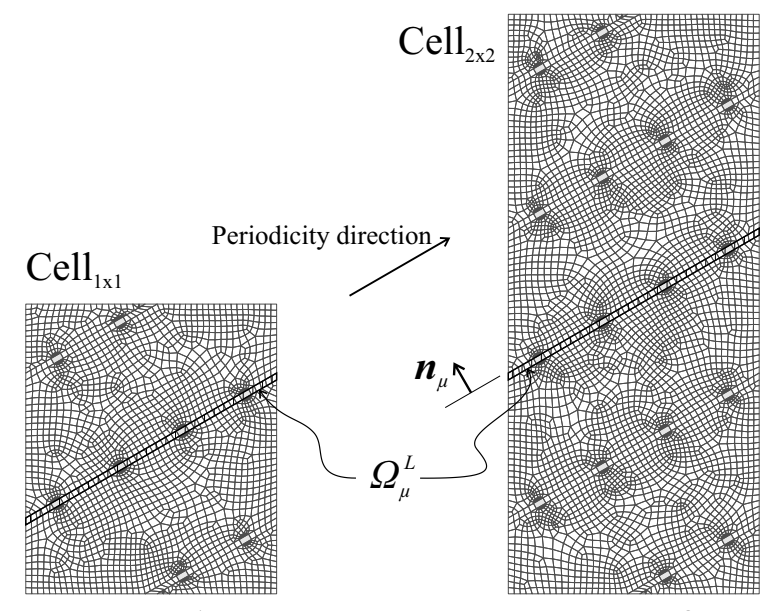

RVE 1

RVE 2

Figure 13: Finite element meshes for the cells: $\mathrm{Cell}_{1 \times 1}$ and $\mathrm{Cell}_{1 \times 2}$. The strain localization domain $\Omega_{\mu}^{L}$ is depicted in both cases.
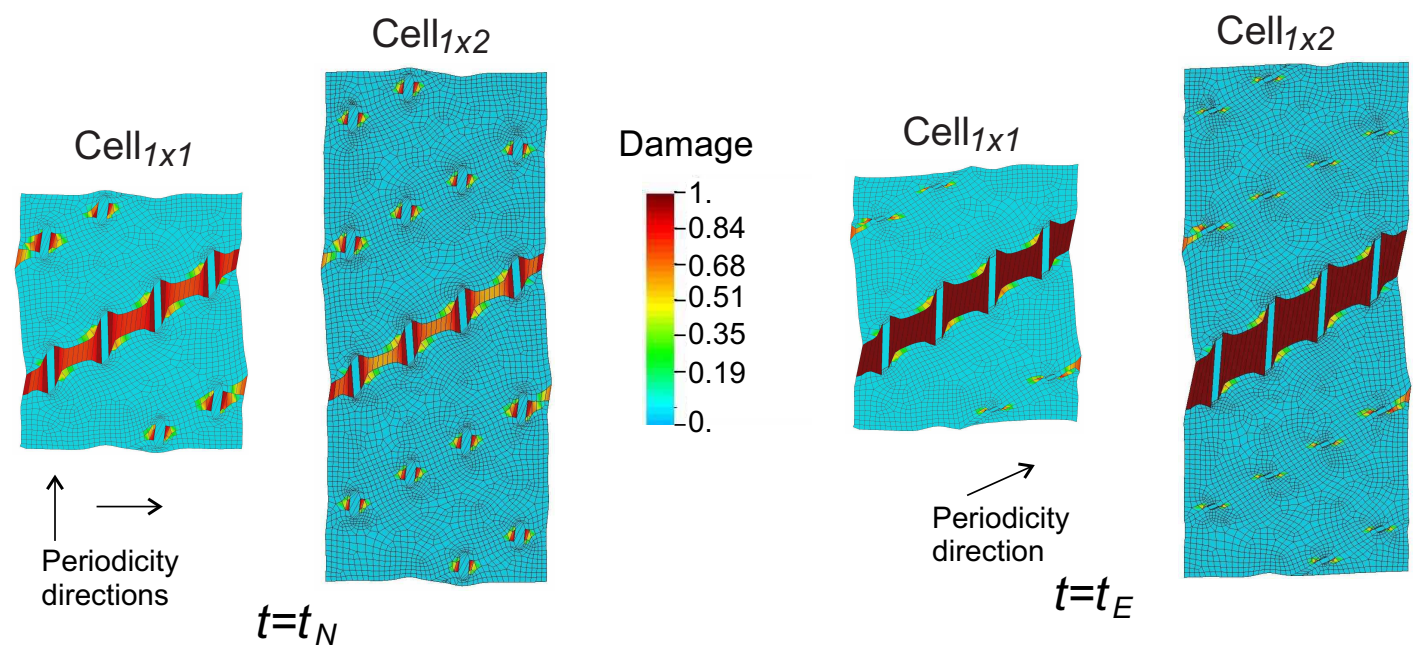

Figure 14: Micro-cell problem with fracture. Cell $1_{1 \times 1}$ and Cell $1_{1 \times 2}$ deformed meshes and damage distribution in two stages of analysis. 


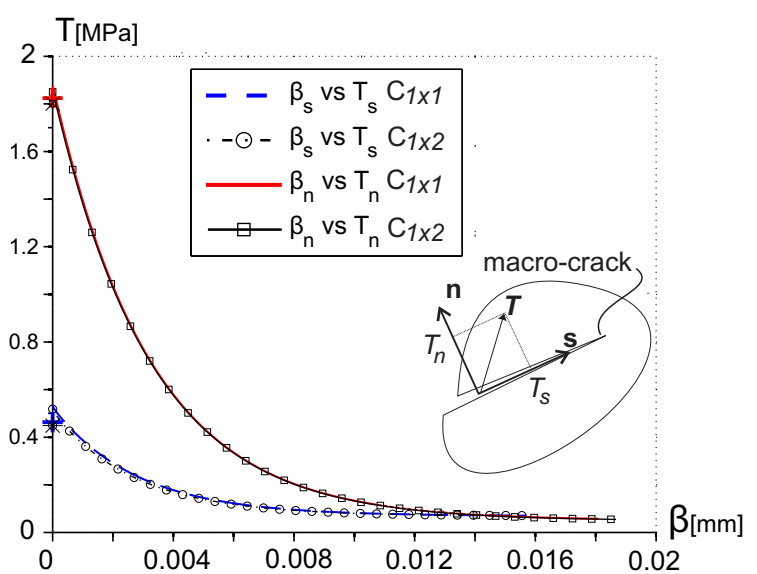

Figure 15: Normal and tangential components of the homogenized traction vector $\boldsymbol{T}$ as a function of the macroscopic displacement jump $\beta$ : Cell ${ }_{1 \times 1}$ and $\mathrm{Cell}_{1 \times 2}$ : periodic sub-model during the stable macroscopic regime.

then, we have determined that, using periodic constraints during the stable regime, with the Cell ${ }_{1 \times 1}$ the error is: $e_{T}=4,14 \%$, and with the Cell $_{1 \times 2}$ the error is: $e_{T}=4,41 \%$.

Note that a macroscopic mixed-mode of fracture is captured by using the FOMF approach.

\subsection{Single-Edge Notched Bending (SENB) tests of a beam with microstructure.}

In this test, we assess the accuracy of the multiscale formulation to estimate the fracture energy at the macroscale resulting from the upscaling of the dissipative mechanisms at the microscopic level. To achieve this goal, we choose a test where the macroscopic fracture energy can be analytically evaluated. In the microstructure, the crack pattern leading to failure is predefined. While, the macrocrack path can be precisely estimated.

The tests consist of a three-point notched beam bending problem, undergoing a vertical displacement that is prescribed in the upper mid-span point. Beams with identical geometries, which dimensions are displayed in Figure 16-e, and with different microstructures are simulated. A plane strain hypothesis is considered and the beam thickness is $1 . \mathrm{mm}$.

\subsubsection{Description of the material and its microstructure.}

Three types of periodic microstructures with a regular arrangement of voids are modeled. Inserts $\mathrm{a}, \mathrm{b}$ and $\mathrm{c}$ in Figure 16 display a detail of the microstructures. The volume fractions of voids $f_{v}$ in each one of the three cases are: 0.0, 0.037 and 0.111 , respectively. A central vertical band of finite thickness, crossing the voids, is characterized with a damage model that degrades under tensile stress states. This material is denoted M1 in Figures 16. This band is embedded into an elastic matrix of material denoted M2 in the Figure. The microvoids are typified by an extremely soft elastic material $(E \rightarrow 0)$ denoted M3. The fact of treating the voids in this way simplifies the algorithmic procedure which is used for detecting the localized domain $\Omega_{\mu}^{L}$, as well as for evaluating the boundary surface $\Gamma_{\mu}^{L}$ where the NSBC should be prescribed, once the CohMM is adopted. Then, a crack is expected to develop in the micro-cells along the vertical band.

The material M4, defined at the macrostructural level, behaves according to the multiscale model, i.e. macro-stresses $\sigma$ depend on macro-strain $\varepsilon$ through the homogenization procedure. While, material M5 is a macroscopic elastic model with a constitutive equation given by:

$$
\dot{\boldsymbol{\sigma}}=\left(\boldsymbol{C}^{e}\right)^{h o m o g} \dot{\boldsymbol{\varepsilon}}
$$

where $\left(\boldsymbol{C}^{e}\right)^{\text {homog }}$ is the homogenized elastic tensor that is obtained from each microstructure, with equation (23), in an elastic loading process. For every microstructure, the tensor $\left(\boldsymbol{C}^{e}\right)^{\text {homog }}$ is determined in a previous micro-cell analysis.

Material parameters for M1, M2 and M3 are presented in Table 2.

\subsubsection{Numerical models.}

Two numerical approaches are used for simulating all these cases: 


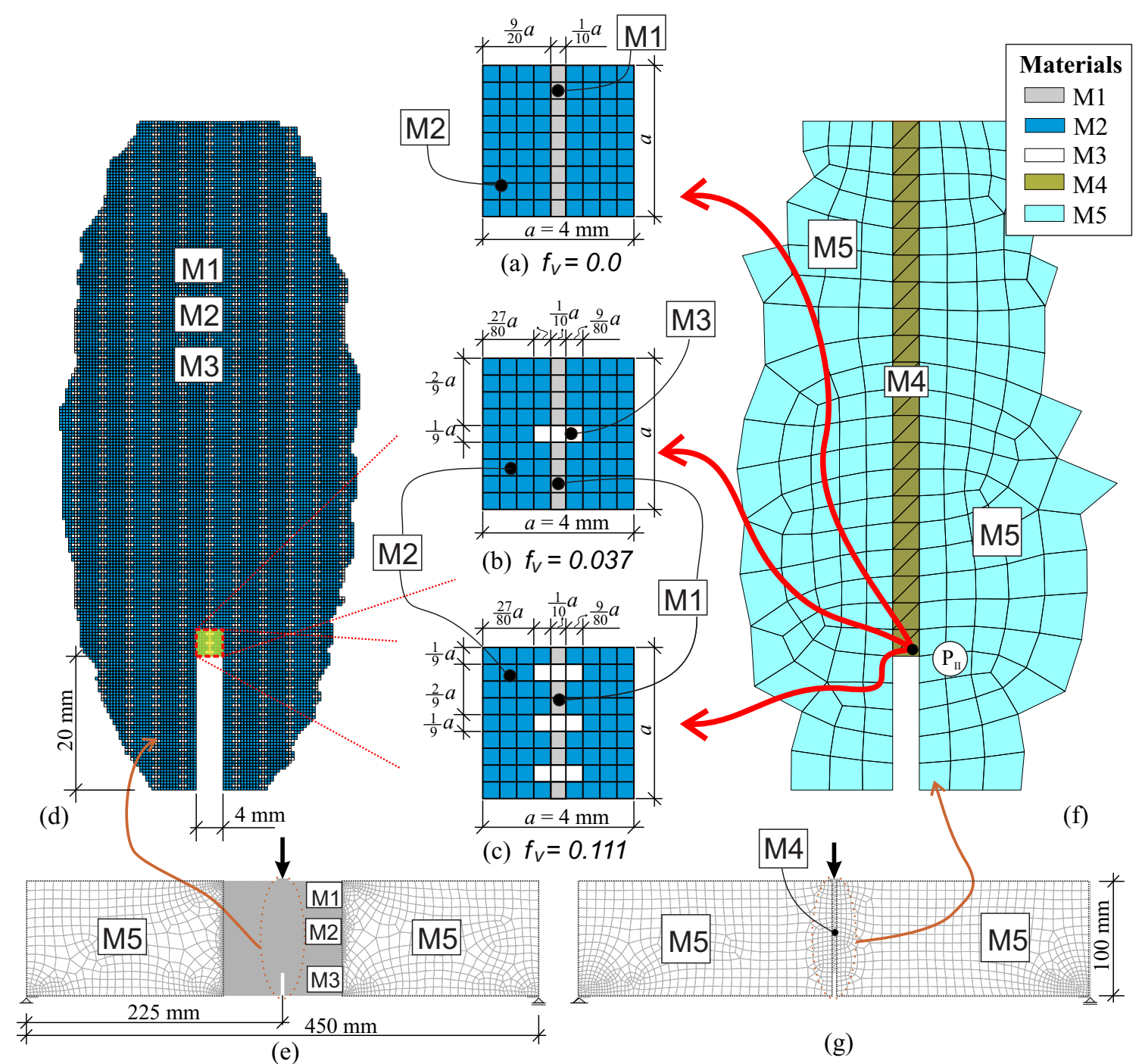

Figure 16: SENB tests and material type distribution in the specimens. a-c) micro-cell models without pores, with one and three pores, respectively, d) DNS models; f-g) MS models; e) specimen geometries.

\begin{tabular}{|c|c|c|c|c|c|c|}
\hline Name & $\begin{array}{c}\text { Constitutive } \\
\text { Model }\end{array}$ & $E$ & $v$ & $G_{f_{\mu}}$ & $\begin{array}{c}\text { Ultimate tensile } \\
\text { stress } f_{c} \\
{[\mathrm{MP}]}\end{array}$ & $\begin{array}{c}\text { Finite element } \\
\text { type }\end{array}$ \\
\hline \hline M1 & $\begin{array}{c}\text { Damage } \\
\text { (microscale) }\end{array}$ & 20 & 0.20 & 100. & 2.4 & Bilinear Quad. \\
\hline M2 & $\begin{array}{c}\text { Elastic } \\
\text { (microscale) }\end{array}$ & 20 & 0.20 & - & - & Bilinear Quad. \\
\hline M3 & $\begin{array}{c}\text { Elastic } \\
\text { (microscale } \\
\text { voids) }\end{array}$ & 0 & 0 & 0. & - & Bilinear Quad. \\
\hline M4 & $\begin{array}{c}\text { Multiscale } \\
\text { (macroscale) }\end{array}$ & - & - & - & - & $\begin{array}{c}\text { SD Linear } \\
\text { Triangle }\end{array}$ \\
\hline M5 & $\begin{array}{c}\text { Elastic } \\
\text { (homogenized } \\
\text { macroscale) }\end{array}$ & - & - & - & - & Bilinear Quad. \\
\hline
\end{tabular}

Table 2: SENB tests with microstructures: material properties and finite element descriptions according to the denomination given in Figure 16. 


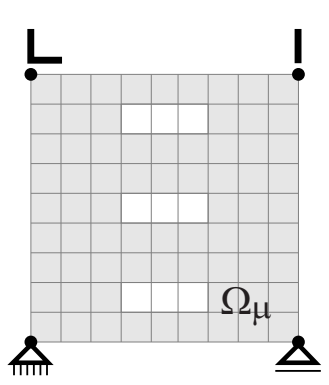

(a) Stable regime

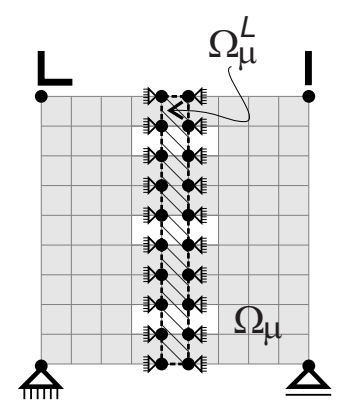

(b) Unstable regime

Figure 17: RVE Boundary conditions. Symbols are defined in Figure 25.

i) Multiscale Simulations (MS), for which the finite element models are shown in Figure 16-g:f and inserts a:c. The beam is modeled with 40 (triangular) E-FEM finite elements in the localization band (material M4) plus 1136 bilinear quadrilateral elements for modeling material M5. The finite element models of the unit cells are depicted in Figure 16-a:c. These cells are simulated with standard bilinear quadrilateral elements. The material softening response is regularized through a smeared crack approach.

ii) Direct Numerical Simulations (DNS). The finite element models of the macro-structure use refined meshes to capture the details of the microstructure. The DNS model only represents the central part of the beam, such as shown in Figures 16-e:d and inserts a:c. The remaining part of the beam is modeled with the material M5. The beam model uses 53703 standard bilinear quadrilateral finite elements. A smeared crack approach regularizing the material strain softening response is taken. The pattern adopted to define the midspan vertical band, where failure is expected to occur, is based on a periodic repetition of microcells, identical in size and geometry, to those used for the RVE's of the MS simulations and shown in Figures 16-a, 16-b and 16-c. Also, the material distributions are identical to those defined for the MS models.

The results obtained with DNS are taken as reference solutions to perform the numerical validation of the MS responses.

In the MS models, and during the macroscopic stable response, the RVE representing the heterogeneous material points (vertical band of the beams) is simulated with a minimum kinematical constraint model, such as that sketched in Figure 17-a. Once detected the bifurcation condition in a macroscopic finite element, the RVE associated to the PGS singular quadrature point, is endowed with the NSBC. In the present case, we adopt a sub-model with linear displacement fluctuation increments in the boundary $\Gamma_{\mu}^{L}$, such as sketched in Figure 17-b. Alternatively, the RVE representing the PGR regular quadrature point, is subjected to the same boundary conditions as that defined in the microcell during the precritical regime, as sketched in Figure 17a. However, the responses of all materials characterizing the RVE of the PGR points are forced to respond elastically during the postcritical regime.

Note that one of the most striking difference between MS and DNS models lies on the fact that, MS models utilize strong discontinuity kinematics for simulating the macroscopic scale. Meanwhile, the DNS models are simulated with a continuum kinematics and the softening response is regularized through a smeared crack model.

\subsubsection{Overall fracture energy obtained with the MS models.}

Figure 18 plots the structural responses of the SENB tests: loads vs. vertical displacements of point $\mathrm{P}_{\mathrm{I}}$. As can be observed, the DNS and MS models furnish almost the same macroscopic solutions for the three microstructures. As expected, microstructures with larger $f_{v}$ require less energy to completely exhaust the structural response.

Furthermore, Figure 19 plots the homogenized traction vs. displacement jump curves which corresponds to the quadrature point where the bifurcation condition is first satisfied (PGS quadrature point located in $\mathrm{P}_{\mathrm{II}}$ of Figure 16-f). The plots of Figure 19 represent the normal components of tractions and displacement jumps. Observe that the crack opening is almost a pure mode I.

Using the plots of Figure 19, we can evaluate the effective density of dissipated energy in point $\mathrm{P}_{\mathrm{II}}$, which is put into play to completely exhaust the macroscopically homogeneous material point. The dissipated 


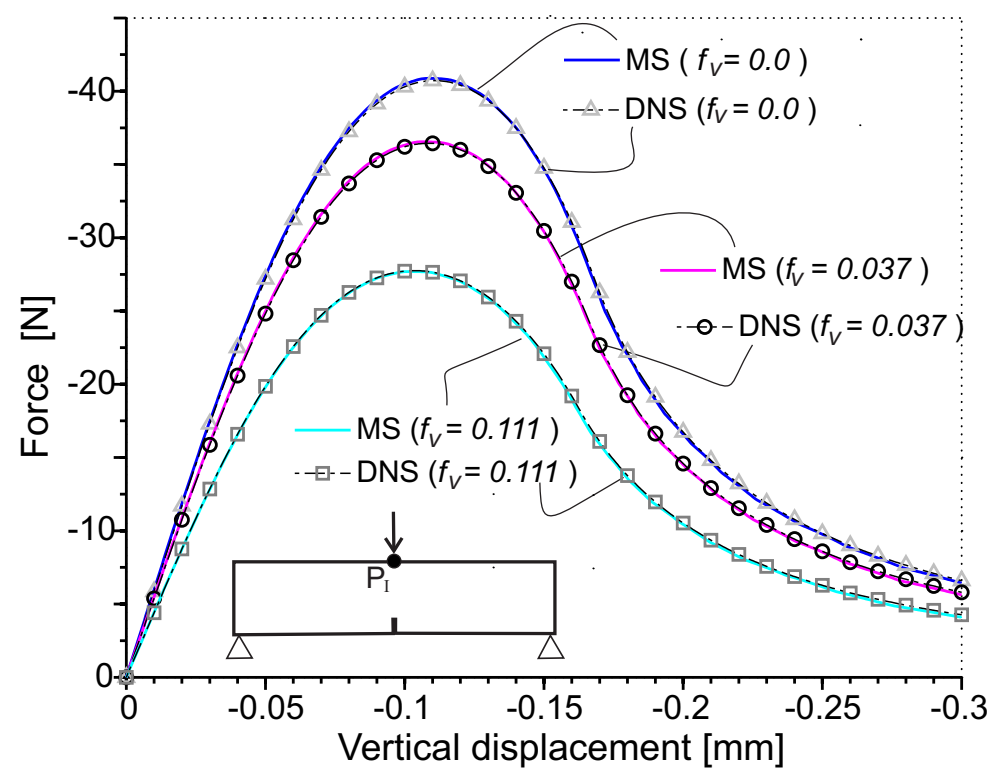

Figure 18: Load vs. vertical displacement of the load application point $\mathrm{P}_{I}$. MS y DNS Models with different void volume fractions.

\begin{tabular}{|c|c|c|c|}
\hline RVE & $\begin{array}{c}\text { Effective Fract. Energy (MS) } \\
G_{f} \\
{[\mathrm{~N} / \mathrm{m}]}\end{array}$ & $\begin{array}{c}\text { Pore ratio } f_{v} \\
\text { (that intersects } \\
\left.\Omega_{\mu}^{L}\right)\end{array}$ & $\begin{array}{c}\text { Fract. Energy estimation } \\
G_{f e} \\
{[\mathrm{~N} / \mathrm{m}]}\end{array}$ \\
\hline \hline$f_{v}=0.0$ & 99.90 & 0 & 100. \\
\hline$f_{v}=0.037$ & 88.42 & 0.1111 & 88.89 \\
\hline$f_{v}=0.111$ & 66.16 & 0.3333 & 66.67 \\
\hline
\end{tabular}

Table 3: SENB test. Effective fracture energy evaluated with different void volume fractions $\left(f_{v}\right)$ in $\Omega_{\mu}^{L}$.

energy is evaluated by determining the area of the plots in Figure 19-a:b, and defining the effective fracture energy:

$$
G_{f}=\int_{t_{N}}^{\infty}(\boldsymbol{T} \cdot \dot{\boldsymbol{\beta}}) d t
$$

This parameter is depicted in Table 3, column 2. Alternatively, we evaluate the energy dissipated by the RVE through the dissipated energy of those finite elements belonging to the localization band $\Omega_{\mu}^{L}$ including voids, and after the macroscopic bifurcation conditions has been detected $\left(t>t_{N}\right)$ :

$$
G_{f e}=\frac{1}{\left|\Omega_{\mu}^{L}\right|} \int_{\Omega_{\mu}^{L}} G_{f_{\mu}} d \Omega
$$

where $G_{f_{\mu}}=100 . \mathrm{N} / \mathrm{m}$ for the material M1 and $G_{f_{\mu}}=0 . \mathrm{N} / \mathrm{m}$ for the material M3, as shown in Table 2 . The energies $G_{f e}$ are shown in Table 3, column 4, for each microstructure. Note the effect that the variable $f_{v}$ has on the effective fracture energy.

Comparing the so obtained values $G_{f}$ and $G_{f e}$, it is observed that, for the RVE without pores, the agreement between both values is almost exact. A slightly larger disagreement is observed when microstructures with one and three pores are analyzed. This result is explained because $G_{f e}$ is determined by assuming that, during the stable regime (i.e. previous to the macroscopic bifurcation detection), energy dissipation has not occurred. In the case of the RVE without pores, the problem is homogeneous before bifurcation because all materials have the same elastic constant. And macroscopic bifurcation detection happens just at the moment that the material band, described with the damage model, reaches the limit strength. Then, the assumption that no dissipation before bifurcation detection happens, is correct. However, in the RVE cases with pores, damage during the stable regime happens.

Thus, the assumption that the fracture energy is evaluated by (64) is no longer correct and the parameter $G_{f e}$ thus evaluated, overestimates the fracture energy of the material M4.

The normal vectors to the macroscopic discontinuity surface, evaluated with the condition (44), are depicted in Figure 19-b. 


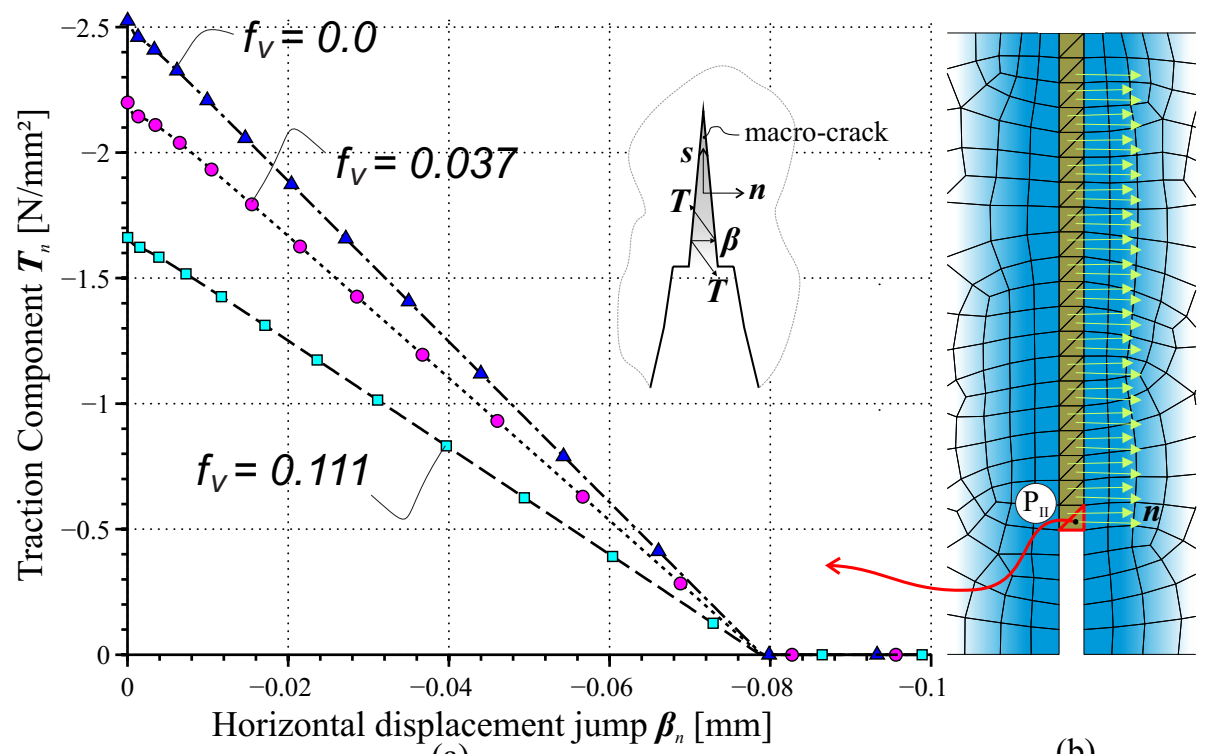

(a)

(b)

Figure 19: MS model. Horizontal-components of the traction vectors vs. horizontal displacement jump evaluated in the first point (picture-b) satisfying the bifurcation condition.

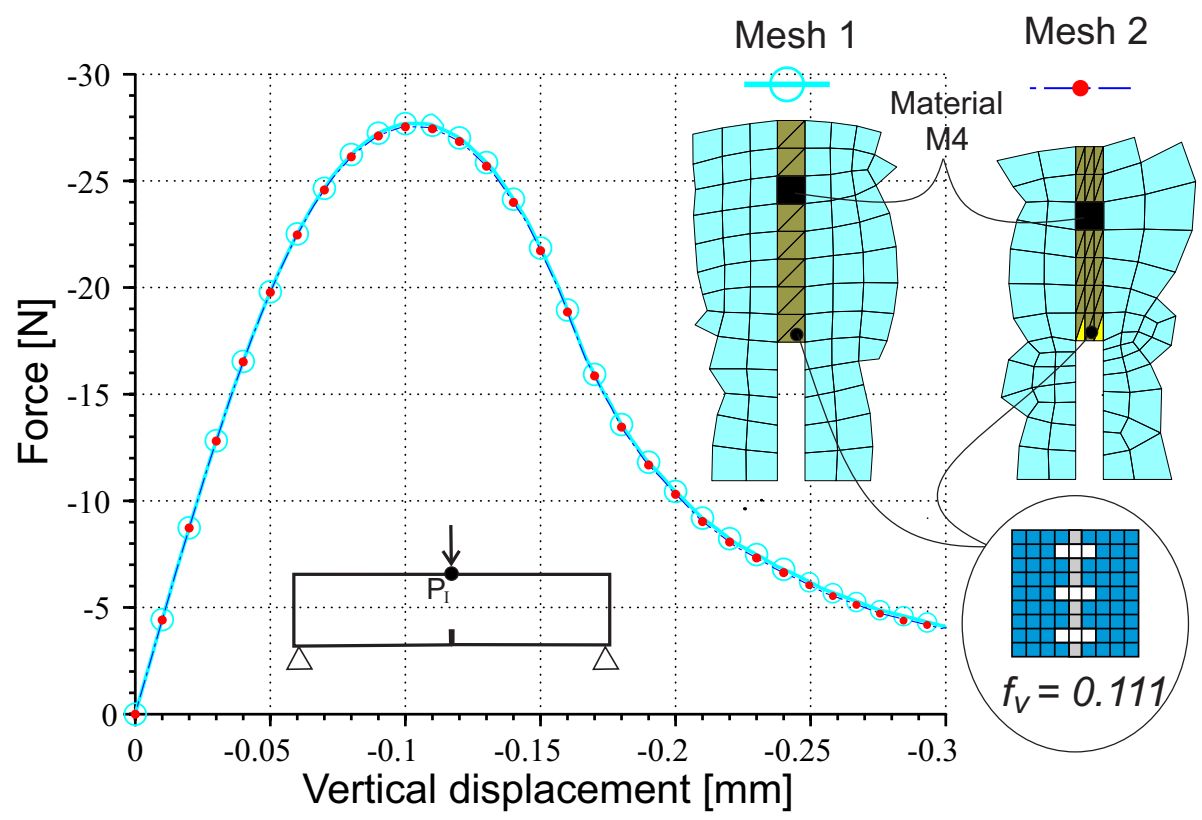

Figure 20: Load vs. vertical displacement of the load application point $\mathrm{P}_{I}$. MS Models with two macroscopic mesh sizes. 

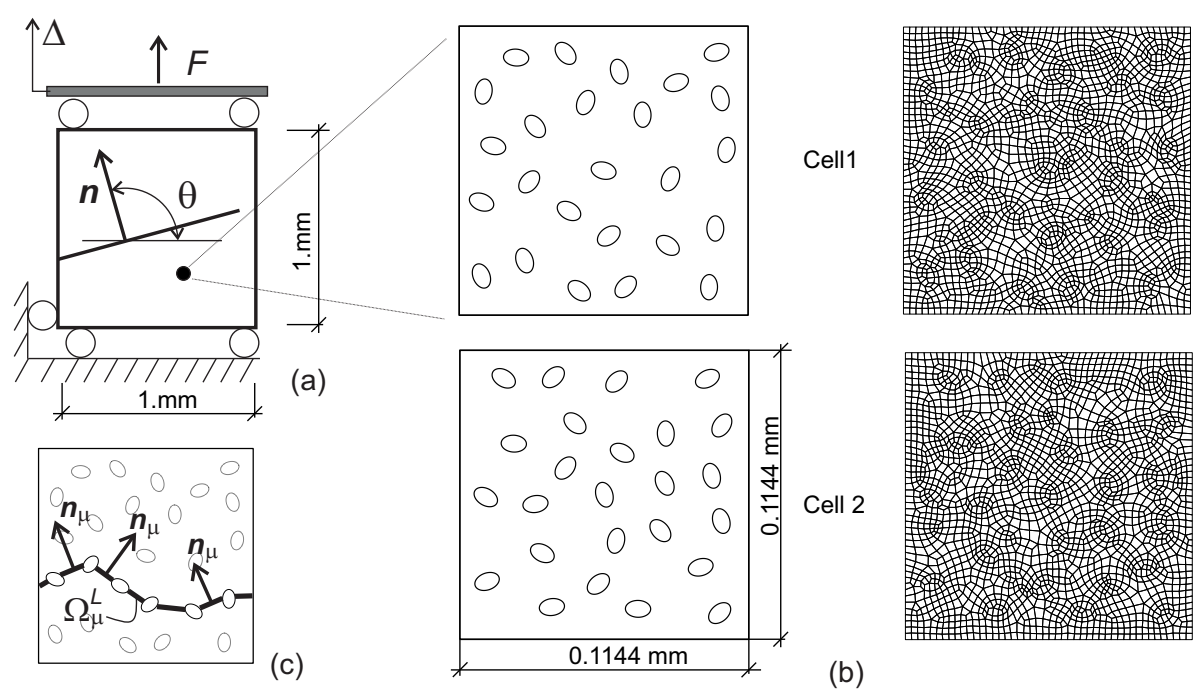

(b)

Figure 21: Strip with a microstructure undergoing uniaxial tensile loading. a) Sketch of the macroscopic test; b) micro-cells 1 and 2; c) normal vectors to the localization band.

Figure 20 shows the objectivity of the structural multiscale response with respect the macroscopic finite element mesh size. The finite element mesh of Case 2 displays smaller elements with respect to Case 1, in the zone where the multiscale material M4 is constitutively simulated.

\subsection{Uniaxial tensile test.}

We simulate the failure of a strip with a microstructure undergoing uniaxial tensile loading in the vertical direction, such as depicted in Figure 21. A vertical displacement $\Delta$ is imposed uniformly on the upper edge of the strip, while the lower edge is fixed.

\subsubsection{Description of the microstructure}

The material is composed of a homogeneous matrix with a statistically uniform distribution of elliptical microvoids embedded in it. All micro-voids have elliptical shapes and are of identical sizes. Their major axes are equal to $0.01[\mathrm{~mm}]$ and the ratio between the major and minor semi-axes is 1.5 . The direction of the major axis of the elliptical pores, as well as the position of the centers, are randomly distributed. The volume fraction of voids is: $f_{v}=0.1$.

The mechanical behavior of the matrix material is described by a damage model that is characterized with the following parameters: Young's modulus: $200 \mathrm{GPa}$, Poisson's ratio: 0.2, Fracture Energy: $0.5 \mathrm{~N} / \mathrm{m}$, and ultimate tensile stress: $2.4 \mathrm{MPa}$. An exponential softening relation is adopted.

As it was explained in previous tests, the voids are also meshed and a soft material is assigned to them with a Young's modulus: $2.0 \times 10^{-9} \mathrm{GPa}$.

\subsubsection{Finite element model}

The strip, at the macro level, is simulated with two E-FEM triangles. In order to compare solutions provided by different micro-cells, we have simulated two cells of identical sizes by changing randomly the distribution and position of voids. In Figure 21, the two cells are denoted: Cell 1 and Cell 2. The meshes of Cells 1 and 2 have 2142 and 2122 bilinear quadrilateral finite elements, respectively.

Periodic standard boundary conditions are assumed, while, for the non-standard boundary conditions, we impose null-fluctuations (Taylor sub-model) in $\Omega_{\mu}^{L}$. In order to inject the strain $d \boldsymbol{\varepsilon}_{\mu}$, given by equation (27), into the micro-cells, it is necessary to determine the normal vectors $\boldsymbol{n}_{\mu}$ to the strain localization domain $\Omega_{\mu}^{L}$. These normal vectors are sketched in the Figure 21-c. And they are evaluated just before inserting the macro-crack, and once the strain localized zone is known.

\subsubsection{Discussion of results}

Figure 22 displays the macrostructural responses of the strip, which are obtained with the micro-cells 1 and 2. We plot the load vs. vertical displacement of the upper edge of the strip. When comparing these 


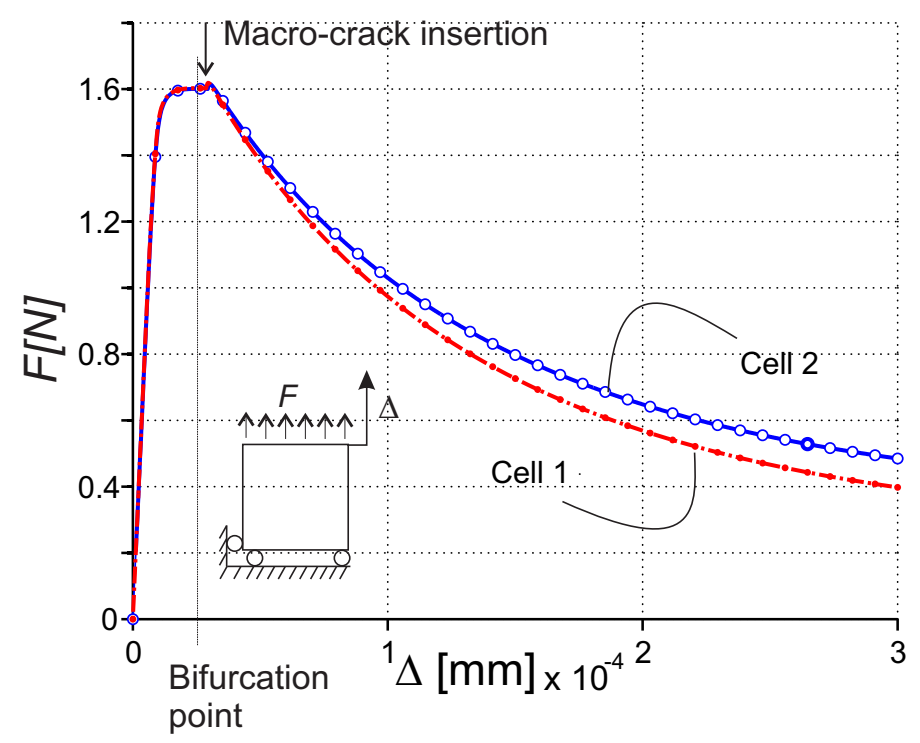

Figure 22: Macrostructural response of the strip with a microstructure undergoing uniaxial tensile loading.

solutions, it is observed a good agreement during the macroscopic stable regime. However, during the postcritical regime, a small difference is observed. We think that this disagreement is caused by the, wellknown, deficient response supplied by the standard bilinear quadrilateral elements for capturing arbitrary localized failure modes. An additional argument in favor of this conclusion can be obtained by observing Figure 24. There, we depict the localized domain that is captured by the cells 1 and 2. The localization bands, using both cells, have more than one finite element width, probably, due to the poor behavior of the finite element.

Because the problem is macroscopically homogeneous, all macroscopic points reach the bifurcation time at the same instant, which is depicted in the plots of the structural response. The bifurcation time has been detected when the vertical displacement of the upper edge of the strip is $\Delta=0.248 \times 10^{-4}[\mathrm{~mm}]$ for Cell 1, and $\Delta=0.254 \times 10^{-4}[\mathrm{~mm}]$ for Cell 2. Also, a few time steps after, we have marked the macrocrack insertion instant. It happens when the vertical displacement is $\Delta=0.288 \times 10^{-4}[\mathrm{~mm}]$ for Cell 1 , and $\Delta=0.294 \times 10^{-4}[\mathrm{~mm}]$ for Cell 2 . The macro-crack insertion coincides with the model change in the microcells. The bifurcation angle detected with the cells are: $\theta=-84.6[\mathrm{deg}]$ for Cell 1 , and $\theta=89.55[\mathrm{deg}]$ for Cell 2.

Figure 23 displays the evolution of the damage distribution that is obtained with the micro-cell 1 . The sequence of pictures temporally agrees with the points marked in the structural response inserted in the same Figure. During the initial stage of material degradation and before the detection of the macroscopic bifurcation, it is observed at the microscopic scale a number of evolving cracks that eventually coalesce into a single micro-crack with a zigzag path.

Figure 24 displays the evolution of the strain localization zone $\Omega_{\mu}^{L}$ for Cells 1 and 2. It is observed that $\Omega_{\mu}^{L}$ changes during the precritical stage. After the bifurcation instant, the strain localization domains remain fixed because the coalescence phenomenon of the micro-cracks.

\section{Conclusions}

A Failure-Oriented Multiscale variational Formulation (FOMF), devised for modeling the connection between failure mechanisms taking place in both macro and micro scales, is presented. Particular attention is paid to the numerical implementation of the model, which is based on the finite element method. We introduce an E-FEM technique for simulating the cohesive surface at the macroscale, and a standard finite element technique at the microscale that regularizes the material response with softening by means of a smeared crack model.

Within the FOMF framework, the kinematical restrictions (boundary conditions) to be applied at the $R V E$-level change, in an incremental sense, during the loading history. In this regard, a generalized and very flexible treatment given to such boundary conditions has been presented in detail throughout the paper. It is mandatory to formulate the problem in rates, which allows for introducing the boundary conditions in an incremental sense. 


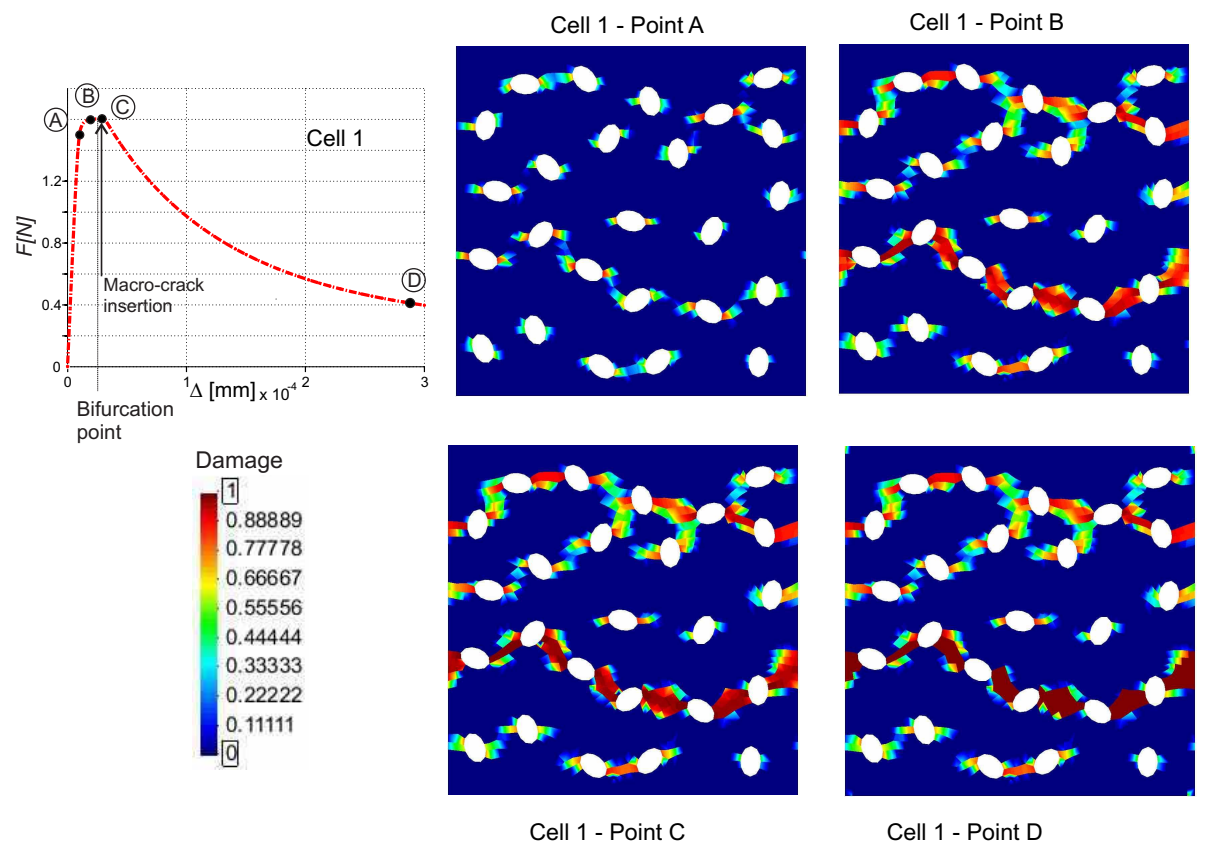

Figure 23: Strip with a microstructure undergoing an uniaxial tensile test. Evolution of the damage distribution for Cell 1.

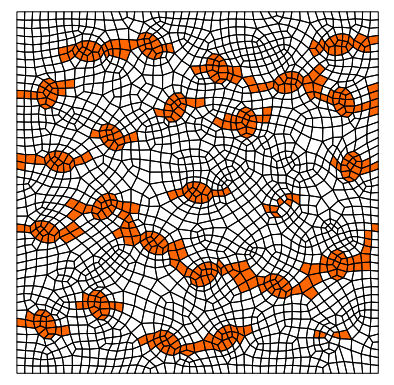

Cell 1 - Point A
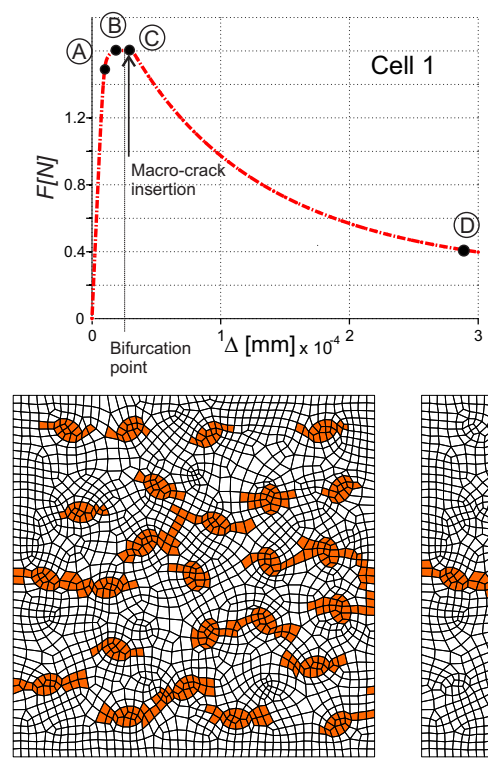

Cell 2 - Point A

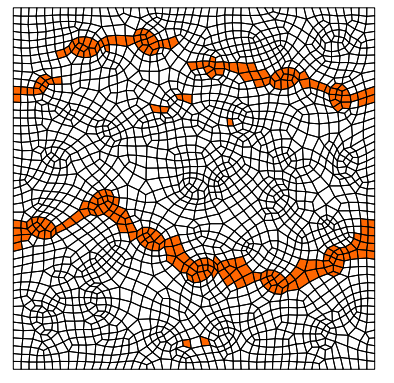

Cell 1 - Point B

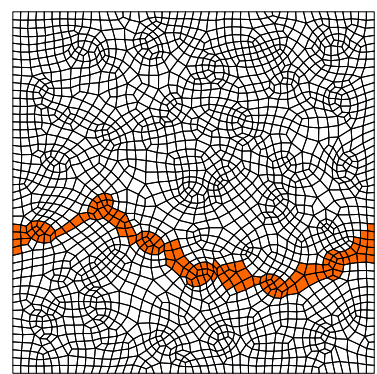

Cell 1 - Points $C$ and D

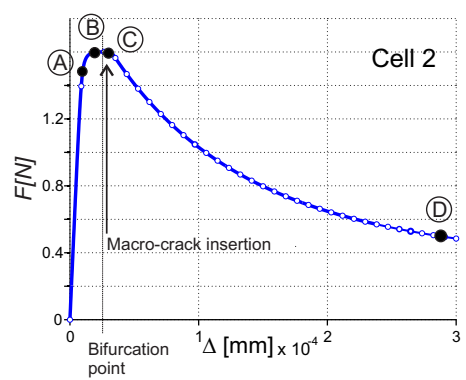

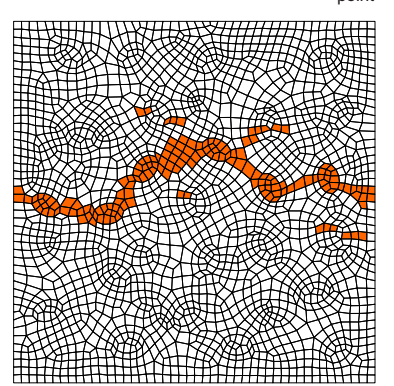

Cell 2 - Point B

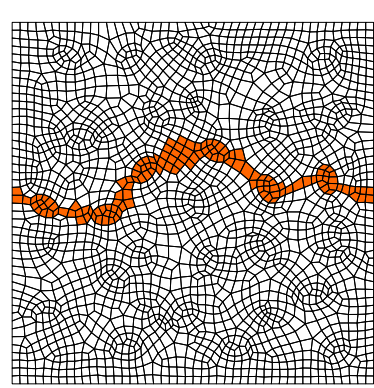

Cell 2 - Points $C$ and D

Figure 24: Strip with a microstructure undergoing an uniaxial tensile test. Evolution of the strain localization domains $\Omega_{\mu}^{L}$ for Cells 1 and 2 . 
In the first numerical test of Section 5, we have evaluated different cell sizes and sub-models in order to understand their effects on the captured failure modes. In this sense, we conclude that different sub-models supply similar results under the condition that the micro-cells are large enough. Also, in the same test, we have evaluated the RVE model capability to reach a complete degradation of the homogenized material without introducing spurious responses. To reach this goal, a very important aspect of the methodology is the introduction, after detecting the stability loss at the macro scale, of the hybrid boundary conditions with periodicity aligned with the crack. This concept was taken from Coenen et al. [6].

As a rigorous proof of the model performance, a comparative study in terms of the Load-Displacement curves (at macro-level) obtained using the FOMF and DNS approach have been conducted for a typical problem in the fracture mechanics context (the three-point bending test). The numerical results show full consistency between both methodologies during the pre and post-bifurcation regimens, as well as when comparing the dissipated energy at the macroscopic level. These conclusions are clearly proven with the structural responses obtained in Figure 18.

According with the last numerical example presented in this contribution, we can conclude that the present formulation can handle rather complex microstructural mechanisms leading to material failure, either during the macroscopic stable regime of the material, as well as during the postcritical regime.

Finally, a very important conclusion of this work, that complements previous results of the authors, is referred to the objectivity displayed by the homogenization procedure with respect to the micro-cell sizes.

\section{Acknowledgment}

The research leading to these results has received funding from the European Research Council under the European Union's Seventh Framework Programme (FP/2007-2013) / ERC Grant Agreement n. 320815 (ERC Advanced Grant Project "Advanced tools for computational design of engineering materials" COMPDES-MAT).

S. Toro, P.J. Sánchez and A.E. Huespe acknowledge the financial support received from ANPCyT and CONICET of Argentina through grants: PICT 2008-1228 and PIP 112-200901-00341. P.J. Blanco and R.A. Feijóo acknowledge the support of Brazilian agencies FAPERJ and CNPq.

The authors are grateful to J. Hernandez, from the Technical University of Catalonia, by the discussion held on the effects of boundary conditions in the RVE analysis.

\section{A Appendix}

Sub-Section 3.2.1 presents the numerical treatment given to the kinematical constraints in order to define different sub-models of the RVE. In this Appendix, we show additional details about the handling and computational implementation of these generic kinds of boundary conditions.

\section{A.1 Partitioning of d.o.f.'s and static condensation.}

The linear system of equations (59)-(61) expresses the most general kinematical constraints that can be considered in the proposed model. Any other kind of boundary conditions, such as those specified in the cases $i$ ) to iii) in Sub-Section 3.2.1, can be treated as particular cases of the hybrid boundary condition, by removing equations from the mentioned equation system. Let us now rewrite (59)-(61) as follows:

$$
\boldsymbol{J} \Delta \boldsymbol{q}=\mathbf{0}
$$

with

$$
\boldsymbol{J}=\left[\begin{array}{c}
\boldsymbol{I}^{c} \\
\boldsymbol{I}^{p}-\boldsymbol{N}_{\mu} \\
\boldsymbol{H}
\end{array}\right]
$$

with $\boldsymbol{J} \in \mathbb{R}^{(m \times n)}$, being $m=n_{c}+2 n_{p}+3$. These equations implicitly define a natural partition of the vector $\Delta \boldsymbol{q}=\left[\Delta \boldsymbol{q}_{d} ; \Delta \boldsymbol{q}_{f}\right]^{T}$, where $\Delta \boldsymbol{q}_{d} \in \mathbb{R}^{m}$ collects all the dependent d.o.f.'s, and $\Delta \boldsymbol{q}_{f}$ collects all the free d.o.f.'s. Using the same sets of d.o.f.'s, the matrix $\boldsymbol{J}$ and the system of equations (66) can be partitioned and rewritten as:

$$
\boldsymbol{J} \Delta \boldsymbol{q}=\left[\begin{array}{ll}
\boldsymbol{J}_{d} & \boldsymbol{J}_{f}
\end{array}\right]\left[\begin{array}{c}
\Delta \boldsymbol{q}_{d} \\
\Delta \boldsymbol{q}_{f}
\end{array}\right]=\mathbf{0}
$$


which allows for a static condensation of the $\Delta \boldsymbol{q}_{d}$ d.o.f.'s, which is given by:

$$
\Delta \boldsymbol{q}_{d}=\boldsymbol{L} \Delta \boldsymbol{q}_{f}
$$

with: $\boldsymbol{L}=-\left(\boldsymbol{J}_{d}\right)^{-1} \boldsymbol{J}_{f}$.

Thus, we can typify the different spaces: $\tilde{\mathscr{U}}_{q}$ and $\mathscr{V}_{q}$, for each RVE sub-model, by means of the following unified notation:

$$
\begin{aligned}
\tilde{\mathscr{U}}_{q} & \equiv\left\{\Delta \boldsymbol{q}=\left[\Delta \boldsymbol{q}_{d}, \Delta \boldsymbol{q}_{f}\right]^{T} \quad \mid \Delta \boldsymbol{q}_{d}=\boldsymbol{L} \Delta \boldsymbol{q}_{f}\right\} \\
\mathscr{V}_{q} & \equiv\left\{\hat{\boldsymbol{q}}=\left[\hat{\boldsymbol{q}}_{d}, \hat{\boldsymbol{q}}_{f}\right]^{T} \mid \hat{\boldsymbol{q}}_{d}=\boldsymbol{L} \hat{\boldsymbol{q}}_{f}\right\}
\end{aligned}
$$

\section{A.2 Selection of dependent nodal d.o.f.'s.}

The selection of the dependent d.o.f.'s of the vector $\Delta \boldsymbol{q}$, should be based on the fact that the static condensation defined in equation (68) can be performed. This requires that the square matrix $\boldsymbol{J}_{d}$ has to be nonsingular. Note that an admissible static condensation of the d.o.f.'s $\Delta \boldsymbol{q}_{d}$ does not automatically guarantee the well-posedness of the finite element micro-cell model, i.e. the non-singularity of the reduced stiffness matrix.

The selection of the dependent nodal d.o.f.'s, $\Delta \boldsymbol{q}_{d}$, is based in the following considerations, see Figure 25:

i) All the d.o.f.'s of the nodes which are prescribed through equations of the type (59), must belong to $\Delta \boldsymbol{q}_{d}$. The partition of the displacement vector associated with these d.o.f.'s, is generically denoted as $\Delta \boldsymbol{q}_{d}^{c}$

ii) All the d.o.f.'s of the nodes in $\left(y^{+}\right)$, which are linked to points $\boldsymbol{y}^{-}\left(\in \Gamma^{-}\right)$through equation (60), belong to $\Delta \boldsymbol{q}_{d}$. The partition of the displacement vector associated with these d.o.f.'s, is generically denoted: $\Delta \boldsymbol{q}_{d}^{p}$.

iii) Every block of equations (61) adds three additional d.o.f.'s to the list of $\Delta \boldsymbol{q}_{d}$. These three d.o.f.'s are associated to nodes in the boundary $\breve{\Gamma}$. The partition of the displacement vector associated with the three d.o.f.'s, is generically denoted: $\Delta \boldsymbol{q}_{d}^{h}$.

Thus, the vector $\Delta \boldsymbol{q}_{d}$ can be partitioned according to: $\Delta \boldsymbol{q}_{d}=\left[\Delta \boldsymbol{q}_{d}^{c} ; \Delta \boldsymbol{q}_{d}^{p} ; \Delta \boldsymbol{q}_{d}^{h}\right]^{T}$. With this partitioning, the equation system (66) can be rewritten as follows:

$$
m\left\{\begin{array}{c}
n_{c}\{ \\
2 n_{p}\{ \\
3\{
\end{array}\left[\begin{array}{ccc|c}
\mathbb{I}^{c} & \mathbf{0} & \mathbf{0} & \mathbf{0} \\
\boldsymbol{J}_{d c}^{p} & \mathbb{I}^{p} & \boldsymbol{J}_{d h}^{p} & \boldsymbol{J}_{f}^{p} \\
\boldsymbol{J}_{d c}^{h} & \boldsymbol{J}_{d p}^{h} & \boldsymbol{J}_{d h}^{h} & \boldsymbol{J}_{f}^{h}
\end{array}\right]\left[\begin{array}{c}
\Delta \boldsymbol{q}_{d}^{c} \\
\Delta \boldsymbol{q}_{d}^{p} \\
\Delta \boldsymbol{q}_{d}^{h} \\
-- \\
\Delta \boldsymbol{q}_{f}
\end{array}\right]=\left[\begin{array}{c}
\mathbf{0} \\
\mathbf{0} \\
\mathbf{0}
\end{array}\right]\right.
$$

It can be observed that the matrix $\boldsymbol{J}_{d}$, in expression (67), corresponds to the square matrix formed by the first $3 \times 3$ sub-block matrices on the left part of equations.

Also, note in the second line of equations (71), that the blocks of three sub-matrices: $\boldsymbol{J}_{d c}^{p}, \boldsymbol{J}_{d h}^{p}$ and $\boldsymbol{J}_{f}^{p}$, contain the negative expressions of the shape functions $\boldsymbol{N}_{\mu}$ evaluated in the point $\boldsymbol{y}^{-}$, as described in the right part of equation (52). Thus, the second block of $2 n_{p}$ equations includes different possible scenarios of prescribing periodic conditions between boundary points. As an example, let us consider a node placed in $\boldsymbol{y}^{+}$and having a displacement $\Delta \boldsymbol{q}^{p}$, see Figure 5 and equation (52). This node is linked to the point $\boldsymbol{y}^{-}$which may not coincide with a finite element mesh node. In this case, the displacement of the point $\boldsymbol{y}^{-}$should be interpolated as shown in the right part of equation (52). This interpolation could involve nodes which: i) are prescribed, ii) belong to the minimum kinematical constraint condition, or iii) are fixed nodes (i.e. prescribed with zero displacement increment). Their respective displacements belong to the blocks denoted $\Delta \boldsymbol{q}_{d}^{c}, \Delta \boldsymbol{q}_{d}^{h}, \Delta \boldsymbol{q}_{f}$. Then, the sub-matrices: $\boldsymbol{J}_{d c}^{p}, \boldsymbol{J}_{d h}^{p} \boldsymbol{J}_{f}^{p}$ will be constituted by the negative expressions of the shape functions associated to those nodes, respectively.

In the third line of (71), the block of three equations (assuming problems in $\mathbb{R}^{2}$ ) are built with matrices $\boldsymbol{H}^{j}$, as shown in equations (55)-(57), and the addition of zero-matrix blocks, depends on which d.o.f.'s, $\Delta \boldsymbol{q}_{d}^{c}$, $\Delta \boldsymbol{q}_{d}^{p}, \Delta \boldsymbol{q}_{d}^{h}$ or $\Delta \boldsymbol{q}_{f}$ are linked through the minimum kinematical constraint (61). 
Linear incremental model

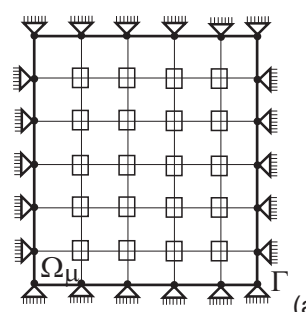

(a)
Periodic incremental model

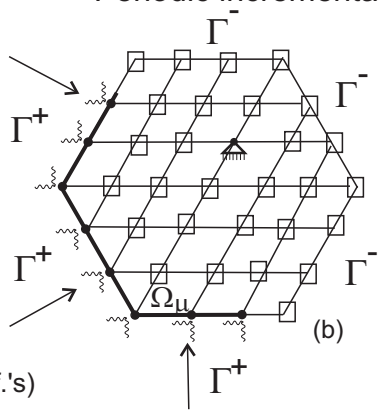

(b)

$\Delta \boldsymbol{q}_{d}^{c}$ 侖 (prescription to zero of two d.o.f.'s)

$d \triangleq$ (prescription to zero of one d.o.f.'s)

$\Delta \boldsymbol{q}_{d}^{p^{m}}$ (horizontal d.o.f. with periodic constraint)

(vertical d.o.f. with periodic constraint)

$\Delta \boldsymbol{q}_{d}^{h}-\quad$ (horizontal d.o.f.'s, minimum kinematical constraint)

$\Delta \boldsymbol{q}_{f} \square$

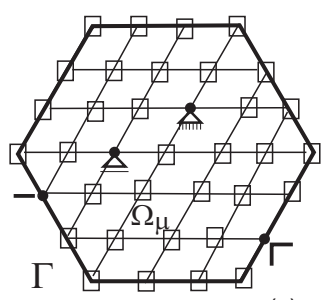

(c)

Minimal kinematic constraint incremental model

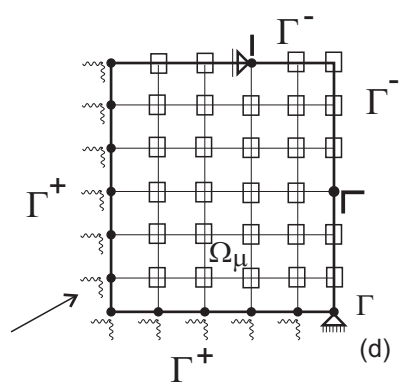

Mixed incremental model

Figure 25: Kinematical constraints defining typical examples of generic RVE sub-models: kind and partition of the d.o.f.'s. a) Linear boundary displacement sub-model; b) periodic sub-model, the arrows indicate the direction of periodicity; c) minimum kinematical constraint model; d) hybrid sub-model. 


\section{A.3 Strategy for solving the finite element governing equations.}

The incremental equilibrium problem of the micro-cell finite element model is written in terms of the displacement fluctuations increments, as follows:

$$
\hat{\boldsymbol{q}}^{T} \cdot \boldsymbol{R}(\Delta \boldsymbol{q})=\hat{\boldsymbol{q}}^{T} \cdot\left[\bigwedge_{e=1}^{n_{\text {elem }}} \int_{\Omega_{\mu}^{e}}\left(\boldsymbol{B}_{\mu}^{e}\right)^{T} \Delta \boldsymbol{\sigma}_{\mu}(\Delta \boldsymbol{q}) d \Omega_{\mu}^{e}\right]=0 ; \quad ; \forall \hat{\boldsymbol{q}} \in \mathscr{V}_{q}
$$

where $\boldsymbol{R}$ represents the residual vector of the incremental internal forces which should be zero; $\bigwedge$ is the standard assembling operator, $n_{\text {elem }}$ the number of finite element in the RVE mesh, $\boldsymbol{B}_{\mu}^{e}$ is the element straindisplacement matrix, $\Omega_{\mu}^{e}$ is the finite element domain in the microscale. We make explicit the dependence of $\Delta \sigma_{\mu}$ with $\Delta q$. In fact, from the material constitutive relation, the microscopic stress increment $\Delta \sigma_{\mu}$ depends on the strain increment: $\Delta \boldsymbol{\varepsilon}_{\mu}:=\Delta \boldsymbol{\varepsilon}+\nabla_{\boldsymbol{x}}^{s}\left(N_{i \mu} \Delta \boldsymbol{q}_{i}\right)$.

The nonlinear system of equations resulting from (72), after inserting the kinematical constraints forcing the finite element functions to belong to (69) and (70), respectively, can be solved through a NewtonRaphson iterative scheme. Then, a typical iteration " $k$ " can be written as follows:

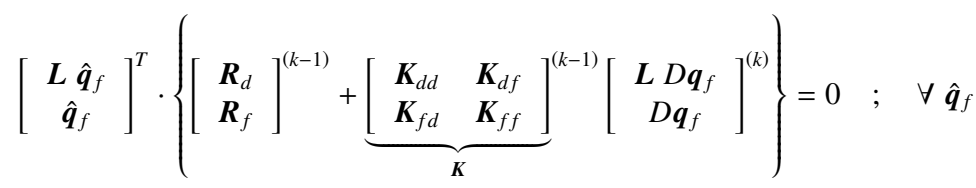

where $\boldsymbol{K}$ is the Jacobian matrix of the residue, $\boldsymbol{K}=\partial \boldsymbol{R} / \partial \Delta \boldsymbol{q}$ and $D \boldsymbol{q}_{f}$ is the increment of $\Delta \boldsymbol{q}_{f}$ at iteration $k$. Expression (73) is partitioned according to the vector partition $\left[\Delta \boldsymbol{q}_{d}, \Delta \boldsymbol{q}_{f}\right]^{T}$ discussed above. Condensing the dependent d.o.f.'s at iteration $k$, the system of equations (73) results:

$$
\left(\boldsymbol{L}^{T} \boldsymbol{K}_{d d} \boldsymbol{L}+\boldsymbol{K}_{d f} \boldsymbol{L}+\boldsymbol{L}^{T} \boldsymbol{K}_{f d}+\boldsymbol{K}_{f f}\right)^{(k-1)} D \boldsymbol{q}_{f}^{(k)}=-\left[\boldsymbol{R}_{f}+\boldsymbol{L}^{T} \boldsymbol{R}_{d}\right]^{(k-1)}
$$

Box 4 shows a pseudo-code written in Matlab, with the implementation of the Newton-Raphson iterative scheme for solving the micro-cell evolution problem. As can be seen, the static condensation procedure is easily implemented by defining two list of d.o.f.'s: the free ones, $d o f_{f}$, and dependent ones $d o f_{d}$, respectively. These d.o.f.'s lists, and also the matrix $\boldsymbol{L}$, may be re-evaluated in every time step that is required by the problem type. The stiffness matrix, $K T$ and the residual vector $\Delta F$ int are assembled using the complete set of d.o.f.'s, and posteriorly, every partition of both terms are selected using the list $d o f_{f}$ and $d o f_{d}$.

The Box 5 shows the pseudo-code of the function that evaluates both lists: $d o f_{f}$ and $d o f_{d}$, and the matrix $\boldsymbol{L}$. 


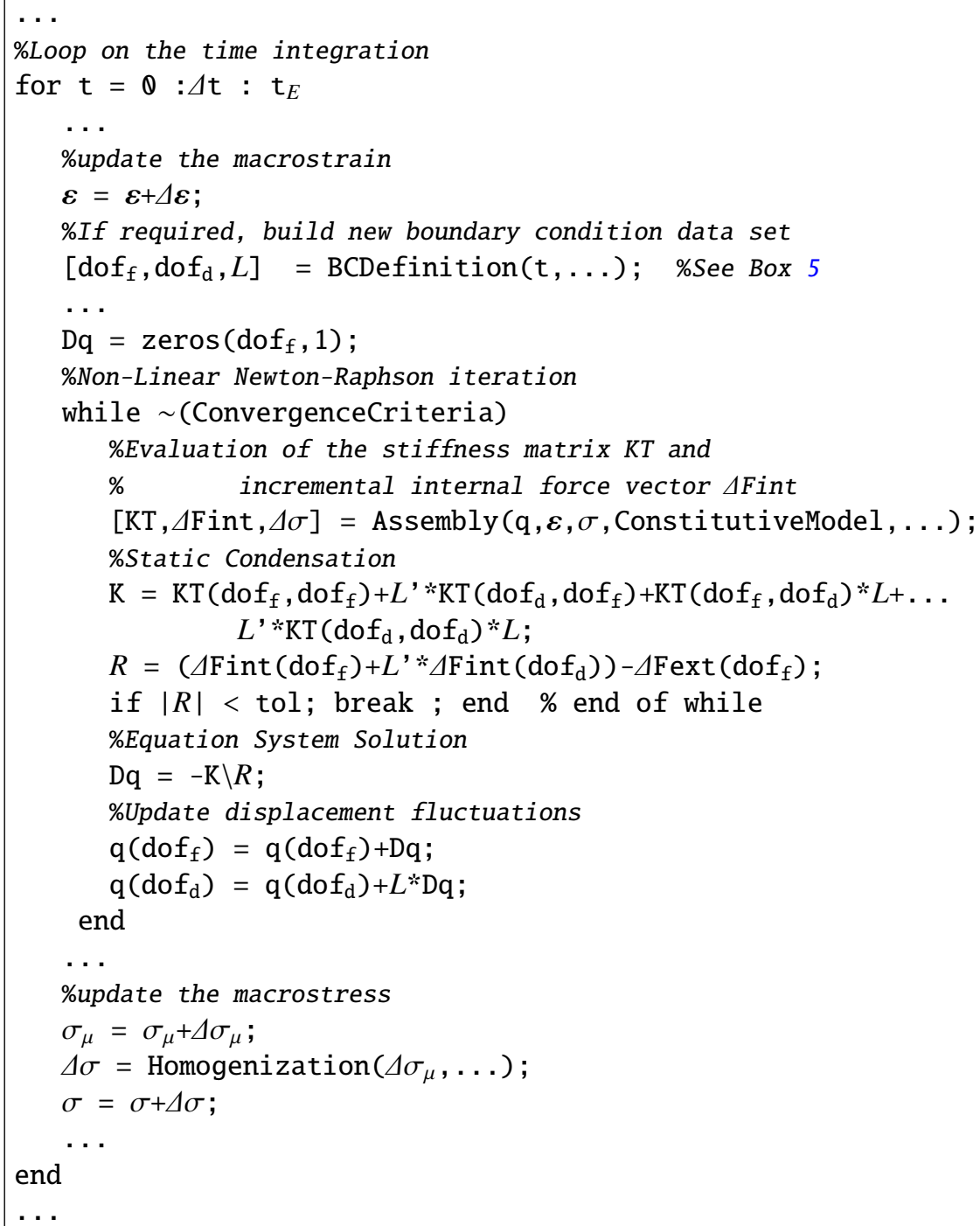

Box 4: Pseudo-code (Matlab) adapted to manage diverse RVE models ( $d o f_{d}$ and $d o f_{f}$ are the list of constrained and free d.o.f.'s, respectively). 


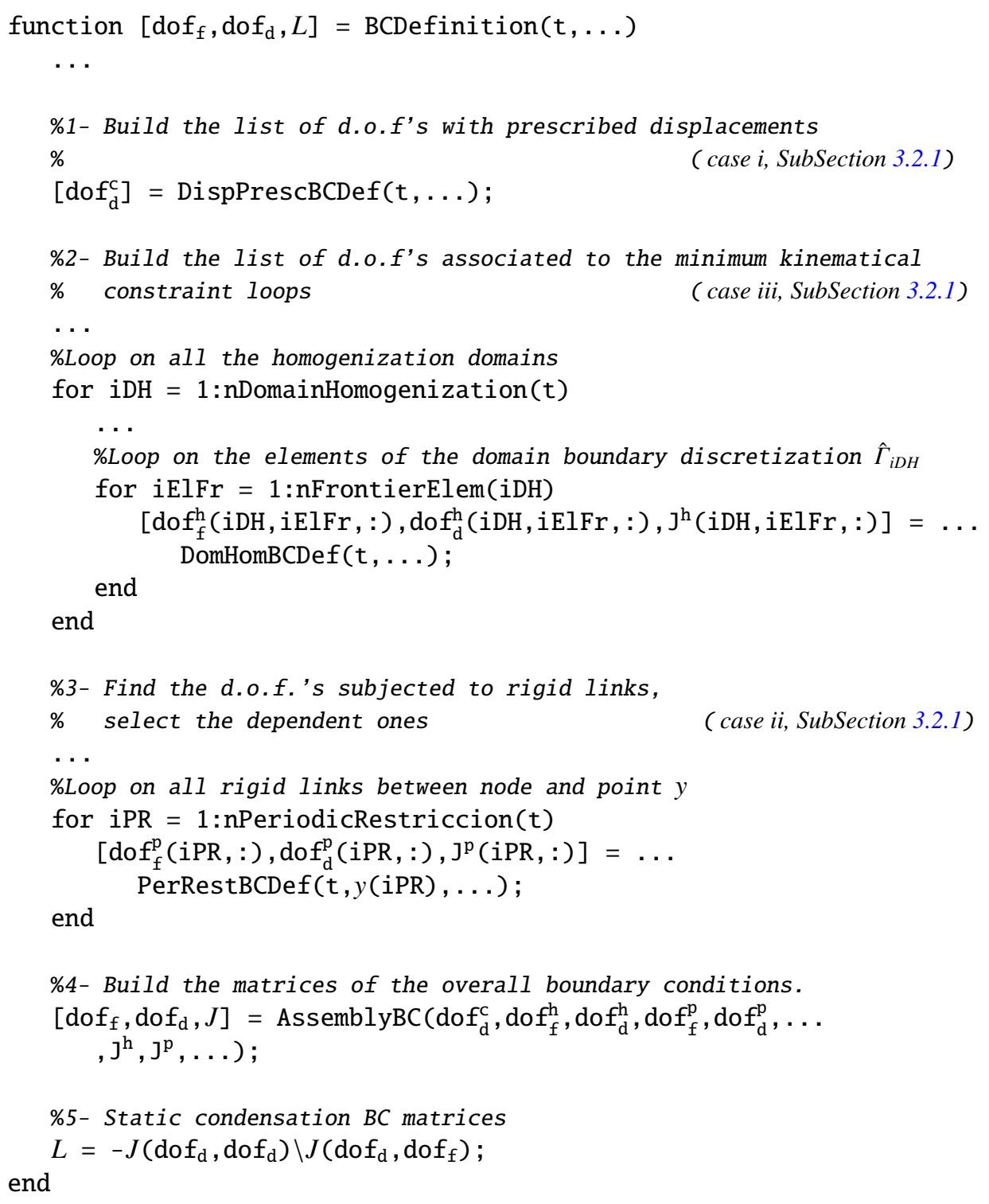

Box 5: Pseudo-code (Matlab). Data-set-definition to manage the RVE boundary conditions ( $d o f_{d}$ and $d o f_{f}$ are the list of constrained and free d.o.f.'s, respectively). 


\section{References}

[1] G. Barenblatt. The mathematical theory of equilibrium of cracks in brittle fracture. Adv. Appl. Mech., 7:55-129, 1962.

[2] Ted Belytschko, Stefan Loehnert, and Jeong-Hoon Song. Multiscale aggregating discontinuities: A method for circumventing loss of material stability. Int. J. Numer. Meth. Engng., 73:869-894, 2008.

[3] A. Bensoussan, J.L. Lions, and G. Papanicolau. Asymptotic analysis for periodic microstructures. North Holland, 1978.

[4] H. J. Böhm. A short introduction to basic aspects of continuum micromechanics. Technical report, The Vienna Institute of Technology: Christian Doppler Laboratory for Functionally Oriented Materials Design, 2008. http://www.ilsb.tuwien.ac.at/links/downloads/ilsbrep206.pdf.

[5] R.M. Christensen and K.H. Lou. Solutions for effective shear properties in three phase sphere and cylinder models. J. Mech. Phys. Solids, 27:315-330, 1979.

[6] E. W. C. Coenen, V. G. Kouznetsova, and M. G. D. Geers. Novel boundary conditions for strain localization analyses in microstructural volume elements. Int. J. Numer. Meth. Engrg., 90:1-21, 2012. DOI: 10.1002/nme.3298.

[7] E. de Souza Neto and R.A. Feijóo. Variational foundation on multi-scale constitutive models of solids: small and large strain kinematical formulation. Technical report, LNCC Research \& Development Report No 16, 2006.

[8] E.A. de Souza Neto and R.A. Feijóo. On the equivalence between spatial and material volume averaging of stress in large strain multi-scale solid constitutive models. Mechanics of Materials, 40:803-811, 2008

[9] J.D. Eshelby. The determination of the field of an ellipsoidal inclusion and related problems. Proc. R. Soc. Lond A, 241:376-396, 1957.

[10] F. Feyel. A multilevel finite element method $\left(\mathrm{FE}^{2}\right)$ to describe the response of highly non-linear structures using generalized continua. Comput. Meth. App. Mech. Eng., 192:3233-3244, 2003.

[11] F. Feyel and J.L. Chaboche. Fe2 multiscale approach for modelling the elastoviscoplastic behaviour of long fibre sic/ti composite materials. Comput. Meth. App. Mech. Eng., 183:309-330, 2000.

[12] J. Fish, Q. Yu, and K. Shek. Computational damage mechanics for composite materials based on mathematical homogenisation. Int. J. Numer. Meth. Engrg., 45:1657-1679, 1999.

[13] M.G.D. Geers, V.G. Kouznetsova, and W.A.M. Brekelmans. Multi-scale computational homogenization: Trends and challenges. Journal of Computational and Applied Mathematics, 234:2175-2182, 2010.

[14] I.M. Gitman, H. Askes, and L.J. Sluys. Representative volume: Existence and size determination. Engineering Fracture Mechanics, 74:2518-2534, 2007.

[15] Z. Hashin. The elastic moduli of heterogeneous materials. J. Appl. Mech., 29:143-150, 1962.

[16] Z. Hashin. Analysis of composite materials. A survey. J. Appl. Mech., 50:481-505, 1983.

[17] Z. Hashin and S. Shtrikman. A variational approach to the theory of the elastic behaviour of multiphase materials. J. Mech. Phys. Solids, 11:127-140, 1963.

[18] R. Hill. A self-consistent mechanics of composite materials. J. Mech. Phys. Solids, 13:213-222, 1965.

[19] A. Hillerborg, M. Modeer, and P. Petersson. Analysis of crack formation and crack growth in concrete by means of fracture mechanics and finite elements. Cement and concrete research, 6(6):163-168, 1976

[20] A.E. Huespe and J. Oliver. Crack models with embedded discontinuities. In G. Hofstetter and G. Meschke, editors, Numerical Modeling of Concrete Cracking, pages 99-159. CISM, Courses\& Lectures 532, SpringerWienNewYork, 2011.

[21] K. Matous, M.G. Kulkarni, and P.H. Geubelle. Multiscale cohesive failure modeling of heterogeneous adhesives. Journal of the Mechanics and Physics of Solids, 56:1511-1533, 2008.

[22] J.C. Michel, H. Moulinec, and P. Suquet. Effective properties of composite materials with periodic microstructure: a computational approach. Comput. Methods Appl. Mech. Engrg., 172:109-143, 1999.

[23] C. Miehe, J. Schotte, and J. Schroder. Computational micro-macro transitions and overall moduli in the analysis of polycrystals at large strains. Computational Materials Science, 6:372-382, 1999.

[24] C. Miehe, J. Schroder, and M. Becker. Computational homogenization analysis in finite elasticity: material and structural instabilities on the micro- and macro-scales of periodics composites and their interaction. Comput. Methods Appl. Mech. Eng., 191:4971-5005, 2002.

[25] T. Mori and K. Tanaka. Average stress in the matrix and average energy of materials with misfitting inclusions. Acta Metall., 21:571-574, 1973.

[26] S. Nemat-Nasser and M. Hori. Micromechanics: overall properties of heterogeneous materials. Elsevier, 1999. 2nd Rev. Edition.

[27] V.P. Nguyen, O. Lloberas-Valls, M. Stroeven, and L.J. Sluys. Homogenization-based multiscale crack modelling: from micro diffusive damage to macro cracks. Computer Methods in Applied Mechanics and Engineering, 200:1220-1236, 2010.

[28] V.P. Nguyen, O. Lloberas-Valls, M. Stroeven, and L.J. Sluys. On the existence of representative volumes for softening quasibrittle materials - a failure zone averaging scheme. Computer Methods in Applied Mechanics and Engineering, 199:3028-3038, 2010

[29] V.P. Nguyen, M. Stroeven, and L.J. Sluys. An enhanced continuous-discontinuous multiscale method for modeling mode-i cohesive failure in random heterogeneous quasi-brittle materials. Eng. Fract. Mech., 79:78-102, 2012.

[30] J. Oliver. On the discrete constitutive models induced by strong discontinuity kinematics and continuum constitutive equations. Int. J. Solids Struct., 37:7207-7229, 2000

[31] J. Oliver and A.E. Huespe. Theoretical and computational issues in modelling material failure in strong discontinuity scenarios. Comput. Meth. App. Mech. Eng., 193:2987-3014, 2004. 
[32] J. Oliver, A.E. Huespe, S. Blanco, and D.L. Linero. Stability and robustness issues in numerical modeling of material failure with the strong discontinuity approach. Comput. Meth. App. Mech. Eng., 195(52):7093-7114, 2005.

[33] J. Oliver, A.E. Huespe, and P.J. Sánchez. A comparative study on finite elements for capturing strong discontinuities: E-FEM vs X-FEM. Comput. Methods Appl. Mech. Engrg., 195(37-40):4732-4752, 2006.

[34] M. Ortiz and A. Pandolfi. Finite-deformation irreversible cohesive elements for three-dimensional crack-propagation analysis. Int. J. Numer. Meth. Engrg., pages 1267-1282, 1999.

[35] D. Peric, E.A. de Souza, R.A. Feijóo, M. Partovi, and A.J. Carneiro Molina. On micro-to-macro transition for multiscale analysis on non-linear heterogeneous materials: unified variational basis and finite element implementation. Int. J. Num. Meth. Eng., 87:149-170, 2011.

[36] M. Pindera, Khatam H, A.S. Drago, and Y. Bansal. Micromechanics of spatially uniform heterogeneous media: A critical review and emerging approaches. Composites: Part B, pages 349-378, 2009.

[37] P.J. Sánchez, P.J. Blanco, A.E. Huespe, and R.A. Feijóo. Failure-Oriented Multi-scale variational formulation for softening materials. Technical Report N 6, LNCC, Petrópolis, R.J., Brasil, 2011.

[38] P.J. Sánchez, P.J. Blanco, A.E. Huespe, and R.A. Feijóo. Failure-Oriented Multi-scale variational formulation: microstructures with nucleation and evolution of softening bands. Comput. Meth. App. Mech. Eng., 257:221-247, 2013. doi: http://dx.doi.org/10.1016/j.cma.2012.11.016.

[39] E. Sanchez-Palencia. Non-homogeneous media and vibration theory. Volume 127 on Lecture Notes in Physics. Springer-Verlag, Berlin, 1980

[40] J. Simo and J. Oliver. A new approach to the analysis and simulation of stong discontitnuities. In Z.B. Bazant, Z. Bittnar, M. Jirásek, and J. Mazars, editors, Fracture and Damage in Quasi-brittle Structures., pages 25-39. E \& FN Spon, 1994.

[41] Jeong-Hoon Song and Ted Belytschko. Multiscale aggregating discontinuities method for micro-macro failure of composites. Composites, Part B 40:417-426, 2009.

[42] F.V. Souza and D.H. Allen. Modeling the transition of microcracks into macrocracks in heterogeneous viscoelastic media using a two-way coupled multiscale model. Int. J. Solids Struct., 48:3160-3175, 2011.

[43] J.F. Unger. An FE ${ }^{2}-\mathrm{X} 1$ approach for multiscale localization phenomena. J. Mech. Phys. Solids, 61:928-948, 2013.

[44] C.V. Verhoosel, J.J.C. Remmers, M.A. Gutiérrez, and R. de Borst. Computational homogenization for adhesive and cohesive failure in quasi-brittle solids. Int. J. Numer. Meth. Engng., 83:1155-1179, 2010.

[45] J.R. Willis. Variational and related methods for the overall properties of composites. Adv. Appl. Mech., 21:1-78, 1981.

[46] X.-P. Xu and A. Needleman. Continuum modelling of interfacial decohesion. Dislocations 93, Solid State Phenomena, 3536:287-302, 1994. 